\title{
Interaction Matter-Polarized Light
}

Yves Joly

\begin{abstract}
This chapter presents the process of interaction of light, mainly in the $\mathrm{x}$ ray range, with matter. The subject is introduced by some experimental evidences of x-ray absorption, emission or scattering by different kinds of material. The ways of describing the electromagnetic wave field are then briefly presented before developing its interaction with an electron in an atom. This process is then applied in the context of core x-ray spectroscopies. The absorption, the dichroism and the resonant diffraction are then specifically presented before giving some key elements to manage monoelectronic simulations.
\end{abstract}

\section{Introduction}

When an electromagnetic wave meets matter, many processes can be observed. The incoming wave can simply be partially -or completely - absorbed by the sample. It can be scattered elastically or inelastically, coherently or incoherently. We can also observe emission of photons at specific wavelengths or of electrons also at characteristic energies or on the contrary on large energy ranges. From the explanation of the photoelectric effect by Einstein, huge progress in the understanding of the physical processes involved in all these phenomena have been done. Moreover enormous advances in the use of these process, often spectroscopies, have been reached. They permit to analyze all kinds of material, solid, liquid or gaseous, ordered or disordered, used in many fields, as biology, geology, chemistry or in condense matter physics. It is impossible to give an exhaustive overview of such a subject. We shall focus mainly in the $\mathrm{x}$-ray regime and consider essentially the absorption and elastic scattering of the photons. To introduce the subject, we first present some of the basic observations. Then, we will give the main concepts of the electromagnetic

Yves Joly

Institut Néel, CNRS and Université Joseph Fourier, BP166 F-38042 Grenoble Cedex 9, France e-mail: yves.joly@grenoble.cnrs.fr 
waves before developing their interaction with matter, first in a microscopic point of view and second using terminology used for the study of dielectrics in order to make the connection between both approaches. The microscopic point of view will concern mainly the transition process between two electronic levels and not the full multi-electronic aspect. The last part will concern the $\mathrm{x}$-ray spectroscopies, mainly absorption including dichroism and resonant diffraction.

\section{Experimental observations of $x$-ray interaction with matter}

Here we give some important measurements showing general aspect connected to the absorption - scattering of x-ray by material. We shall see that the absorption depends on energy, polarization, chemical specie and on the geometrical environment.

\subsection{Absorption}

From its discovery by Roentgen in 1896, the most important use of x-ray comes probably from its faculty to cross matter with different absorption rates depending on its constituents. It is thus an exceptional tool to investigate the structure and the composition of all class of samples. For an homogeneous isotropic material, there is a simple exponential decrease of the transmitted beam intensity $I$ in term of the sample thickness $D$ :

$$
I=I_{0} \exp (-\mu D)
$$

where $I_{0}$ is the incident intensity. This equation, equivalent to the Beer-Lamber law $\left(\ln \left(I_{0} / I\right)=\mu D\right)$, introduces the total linear absorption coefficient $\mu$. This coefficient, for a crystalline solid, is related to the absorption cross-section, $\sigma_{i}$, of the $n$ different chemical elements of the unit cell [16]:

$$
\mu=\frac{1}{V} \sum_{n}^{i=1} \sigma_{i} .
$$

where $V$ is the volume of the unit cell. Most often, it is the absorption cross-section which is used. It is usually expressed in square centimeter or in mega barn (1 Mbarn $=10^{-18} \mathrm{~cm}^{2}$ ).

\subsection{Dependence on energy}

When measuring the energy dependence of the photoelectric absorption crosssection for the interaction of x-rays with material, one can see a decrease from some 
Fig. 1 X-ray absorption spectra of Au metallic foil in a $2.5 \mathrm{Kev}$ energy range. The experiment by Proux and Hazemann[41] shows the abrupt increases in the absorption cross-section at typical value of the chemical element present in the sample and called edges. See the so-called EXAFS oscillations after each edge. Note also that the $\mathrm{L}_{I I I}$ edge is two times higher than the $\mathrm{L}_{I I}$ edge.

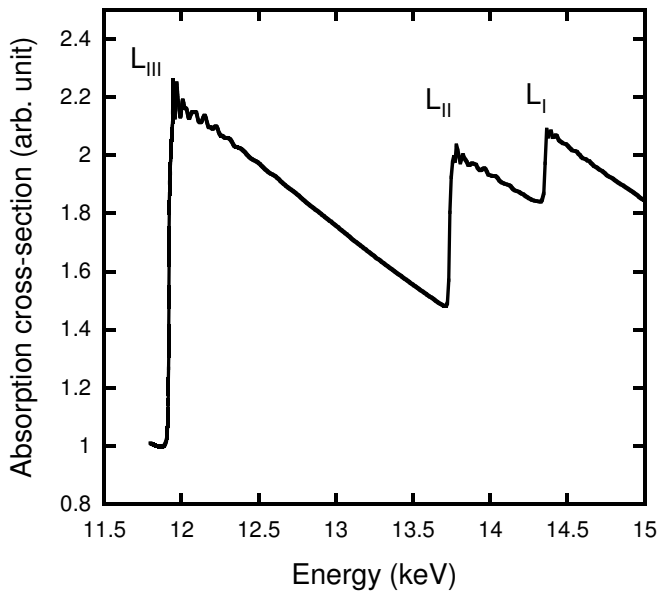

Mbarn at low energy down to some barns at around $1 \mathrm{MeV}$ [3]. At some energies, there are strong and brusque increases of the cross-section. For example in Fig. 1, we show the 11.8 - $15 \mathrm{keV}$ energy range for a gold metallic foil [41]. One can see three edges labeled $\mathrm{L}_{I}, \mathrm{~L}_{I I}$ and $\mathrm{L}_{I I I}$. Note also the oscillations after the edges which are very reach in information on the close neighborhood of the absorbing atoms as it will be seen further on.

Fig. 2 X-ray absorption spectra at different edges of Uranium in Uranyl $\left(\mathrm{UO}_{7}\right)$. All the edges are shifted by the value of the ionization energy of the corresponding edge in order to compare their shapes. The $\mathrm{L}_{I I I}$ and $\mathrm{M}_{I I I}$ are step like and very similar. The other edges have a very different shape. This difference is due to the selection rule imposed on the electronic transition.

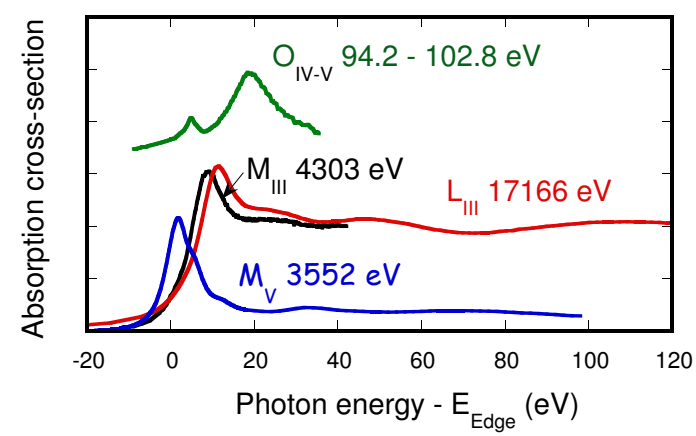

When looking the different edges of a same element in the same material, as for instance the Uranium edge in Uranyl $\left(\mathrm{UO}_{7}\right)$ (Fig. 2) in the experiment performed by Ch. Den Auwer et al. [20], we can see the different kinds of shape of the edges. In this case, we see that the $\mathrm{L}_{I I I}$ and $\mathrm{M}_{I I I}$ have very similar step-like shape. On the other side the $\mathrm{M}_{V}, \mathrm{O}_{I V}$ and $\mathrm{O}_{V}$ present only a single peak, more or less wide. We shall see, Sect. 4.6, that this is due to selection rules in the transition. Another 
observation which is often done is that, as in the Fig. 1, the $\mathrm{L}_{I I I}$ edges is twice higher than the $\mathrm{L}_{I I}$ edge.

The energy of the edges are a signature of the chemical specie of the absorbing atom. A closer look shows that small shifts up to some $\mathrm{eV}$ can be observed in the threshold energy for a same element but in different chemical environments. When increasing the oxidation, the edge tends to shift toward higher energy. This fact is often used to check the valence state of a metal atom in an oxide.

\subsection{Dependence on the atomic environment}

Now let us have a look on the oscillations after a typical edge. We compare two different surroundings of a same chemical specie, as for example the copper K-edge in pure copper metal and in $\mathrm{YCuO}_{0.25}$. In Fig. 3 we can observe the very different oscillation shapes. From other measurements in gas phase or in disordered material we also know that these oscillations do not need a long range order. They are thus a signature of the close environment of the absorbing atoms. The connection between the spectra shape and the neighborhood will be seen in Sect. 6 .

Fig. 3 X-ray absorption spectra at the K-edge of copper in (top) $\mathrm{Cu}$ metallic foil and (down) in $\mathrm{YCuO}_{0.25}$. The edges are roughly at the same energy, but the oscillations are very different.

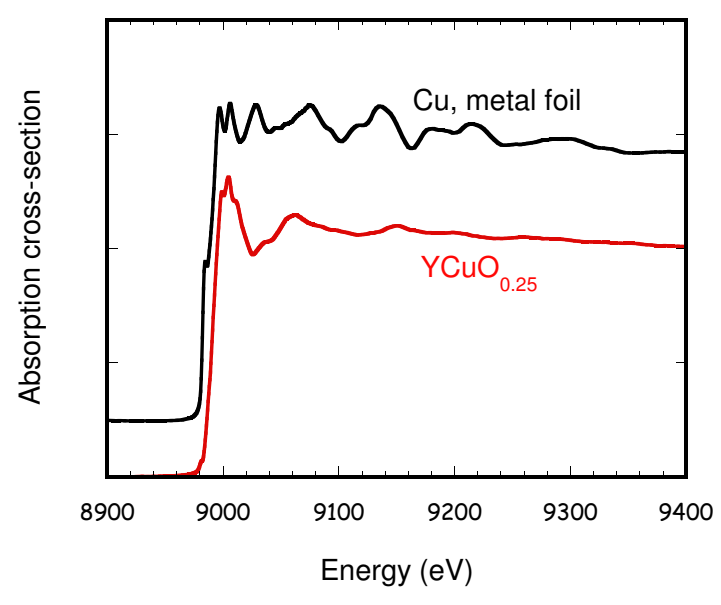

\subsection{Dependence on the polarization light}

Pleochroism or dichroism is the change in color when a mineral is rotated under plane-polarized light. Due to adsorption of particular wavelengths of light, trans- 
mitted light appears colored depending on the thickness and the particular chemical and crystallographic nature of the mineral.

By extension, the difference of absorption, under rotation only, is called dichroism. This phenomena is also observed in the $\mathrm{X}$-ray energy range. To observe this, one needs a single crystal of a non cubic material (note that very small dichroism can also be observed in cubic material). For example, Poumellec et al. [40] measured the absorption along three orientations of a $\mathrm{TiO}_{2}$ single crystal, two are shown in Fig. 4. The effect of the tetrahedral symmetry of the material can be easily verified.

Fig. 4 X-ray absorption spectra of rutile $\mathrm{TiO}_{2}$ around the K-edge of Titanium. The experiment by Poumellec et al. [40] is performed using a single crystal under two orientations of the sample in front of the incoming linear wave field. The difference shown in the figure is called linear dichroism and is relatively strong when the sample is not cubic.

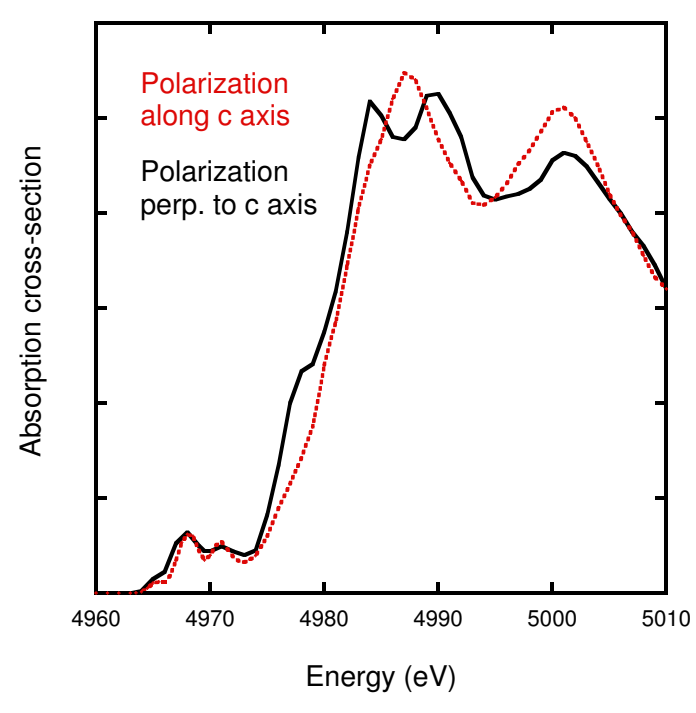

These studies are performed with linear polarized light (the concept of linear or circular polarized light are given in the Sect. 3). In the visible light as well as in the x-ray regime, circular polarized light gives also useful informations. Special interest comes from experiments where one looks at the difference between two spectra recorded respectively with left and right circular polarization. At the $\mathrm{L}_{I I, I I I}$ edges of magnetic material the dichroic signal can reach several percent of the total absorption edge. For example at the Er $\mathrm{L}_{I I I}$ edge in ErZn (see Fig. 5) we see an oscillating behavior around the rising edge energy. At the K-edges, on the contrary, this kind of observations are always very small.

The explanation of these polarization phenomena comes from the dependence of the absorption spectroscopies on the electronic structure around the absorbing atoms. This is detailed in Sect. 6. 
Fig. 5 X-ray absorption spectra of ErZn and the circular dichroic signal at the rare earth $\mathrm{L}_{I I I}$ edge. The latter is a signature of magnetism on the Er atom. The experiment were performed at the ESRF ID12 beam line by Galera $e t$ al. [23]

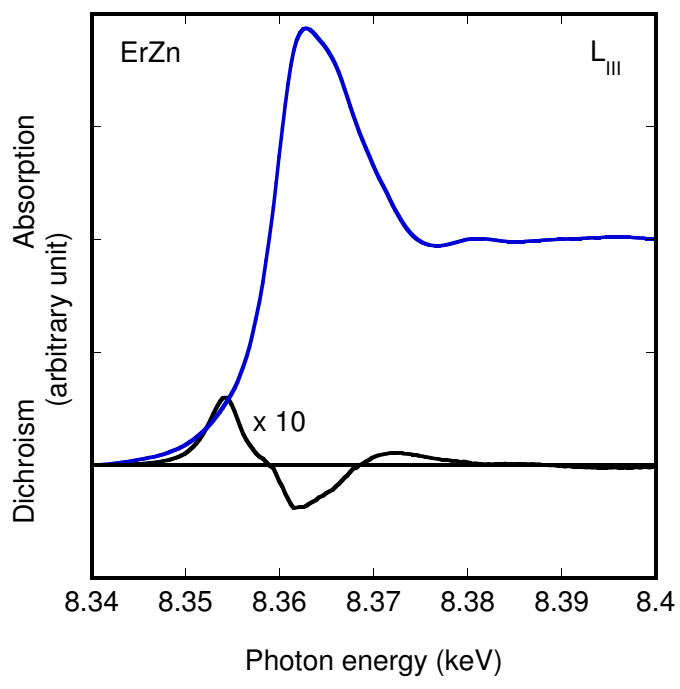

\subsection{Diffraction around edges}

Another kind of experiment increasingly popular at the different synchrotron facilities concerns the measurement of diffracted peaks in a more or less wide energy range around some edges. The technique called diffraction anomalous fine structure (DAFS) or resonant X-ray scattering (RXS) [8] gives set of spectra rich with information on the crystalline material studied. For example in Fig. 6 are shown two spectra of diffraction peaks in $\mathrm{NaV}_{2} \mathrm{O}_{5}$ compared with two absorption spectra measured for two orientations of the sample. The diffracted intensity becomes highly energy dependent around the absorption edges. In some cases, the intensity is nearly zero, but close to the edge, where the variation of intensities have similarities with what is observed in the absorption spectra. This spectroscopy is indeed very close to the absorption spectroscopy, and it is explained for the photon-matter interaction process in Sect. 4 and in an unified scheme to get the final amplitudes in Sect. 6 .

\section{The light}

Before considering the interaction of light with matter, one has to recall the basic definitions used to describe the electromagnetic waves. 
Fig. 6 Two diffraction peaks measured by S. Grenier $e t$ al. [25] around the Vanadium $\mathrm{K}$-edge in $\mathrm{NaV}_{2} \mathrm{O}_{5}$ at $13 \mathrm{~K}$. Their intensity is nearly zero far from the edge and shows strong variations around the edge. They also strongly depend on the polarization conditions. For comparison the absorption spectra is also shown for two linear polarizations. The pre-peak visible in this spectra is also present but eventually slightly shifted in the diffraction spectra.

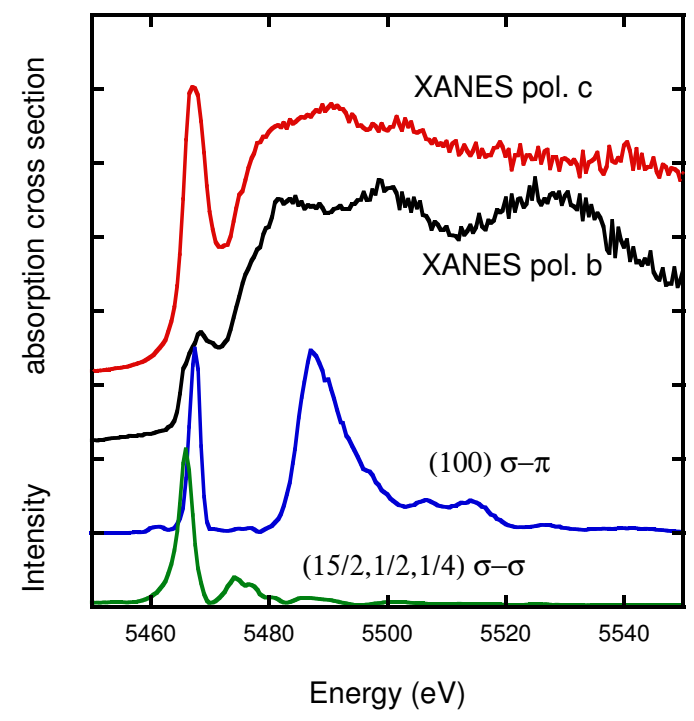

\subsection{Definitions and notations}

The vector potential, A, the electric and magnetic field, $\mathbf{E}$ and $\mathbf{B}$ of the electromagnetic waves are obtained from the Maxwell equations. These ones give first the propagation equations in vacuum:

$$
\Delta \mathbf{A}=\varepsilon_{0} \mu_{0} \frac{\partial^{2} \mathbf{A}}{\partial t^{2}}, \Delta \mathbf{B}=\varepsilon_{0} \mu_{0} \frac{\partial^{2} \mathbf{B}}{\partial t^{2}}, \Delta \mathbf{E}=\varepsilon_{0} \mu_{0} \frac{\partial^{2} \mathbf{E}}{\partial t^{2}} .
$$

A plane wave is a wave with infinitely long and wide wavefront. From the propagation equations it can be shown that in this case:

- the vector potential depends only on time $(\mathrm{t})$ and coordinate corresponding to the propagation direction $\mathrm{Z}$,

- $\mathbf{A}, \mathbf{E}$ and $\mathbf{B}$ are perpendicular to the propagation direction,

- $\mathbf{E}$ and $\mathbf{B}$ are perpendicular to each other.

- $E=c B$

The solutions of the propagation equations are necessarily of the form:

$$
s(z, t)=f(z-c t)+g(z+c t),
$$

where $\mathrm{f}$ and $\mathrm{g}$ are scalar functions.

Progressive plane waves are such that $\mathrm{f}$ or $\mathrm{g}$ is always zero. That is:

- $s(z, t)=f(z-c t)$ is a traveling plane wave propagating along $\mathrm{z}$,

- $s(z, t)=g(z+c t)$ is a traveling plane wave propagating along -z. 
Monochromatic traveling plane waves can be written as :

$$
\begin{aligned}
& A_{x}(z, t)=A_{0 x} \cos \left[2 \pi v\left(\frac{z}{c}-t\right)+\varphi_{0 x}\right], \\
& A_{y}(z, t)=A_{0 y} \cos \left[2 \pi v\left(\frac{z}{c}-t\right)+\varphi_{0 y}\right],
\end{aligned}
$$

where the amplitudes $A_{0 x}$ and $A_{0 y}$ and the phases $\varphi_{0 x}$ and $\varphi_{0 y}$, can have peculiar relations.

Linearly polarized plane waves have both components in phase, that is $\varphi_{0 x}=\varphi_{0 y}$. $\mathbf{E}, \mathbf{B}$ and $\mathbf{A}$ are thus in phase. $\mathbf{E}$ and $\mathbf{A}$ are moreover collinear.

Circularly polarized plane waves are such that $A_{0 x}=A_{0 y}$ and $\varphi=\varphi_{0 y}-\varphi_{0 x}=$ $\pm 90^{\circ}$. The sign plus and minus are respectively for left and right circular polarizations.

Complex notation are more tractable. $\mathbf{A}, \mathbf{E}$ and $\mathbf{B}$ are then given by:

$$
\mathbf{A}=\mathbf{A}_{0} e^{i(\mathbf{k} \cdot \mathbf{r}-\omega t)}, \quad \mathbf{E}=\mathbf{E}_{0} e^{i(\mathbf{k} \cdot \mathbf{r}-\omega t)}, \quad \mathbf{B}=\mathbf{B}_{0} e^{i(\mathbf{k} \cdot \mathbf{r}-\omega t)}
$$

where $\mathbf{k}$ is the wave vector which is collinear to the propagation direction and $\omega=$ $2 \pi \nu$ is the pulsation. Moreover we have the relations: $\mathbf{E}=-i \omega \mathbf{A}, \mathbf{B}=-i \mathbf{k} \times \mathbf{A}$ and $k^{2}=\omega^{2} / c^{2}$

In this context we define the polarization vector by:

$$
\varepsilon=i \frac{\mathbf{E}_{0}}{E_{0}} .
$$

The polarization vector of a circularly polarized wave propagating along $\mathrm{z}$ is thus :

$$
\varepsilon=\frac{1}{\sqrt{2}}\left(\begin{array}{c}
1 \\
\pm i \\
0
\end{array}\right)
$$

In diffraction, the terms of $\sigma$ (or $s$ ) and $\pi$ (or $p$ ) polarizations are often used. They designate the orientation of the polarization, versus the scattering plane. When the polarization is perpendicular to the scattering plane it is called $\sigma$; when it is parallel it is called $\pi$. In classical diffraction, most of the intensity of the diffraction peaks is obtained in the $\sigma$ in $-\sigma$ out channel (one says directly $\sigma-\sigma$ ). With magnetism or/and around the absorption edge one can get important $\sigma-\pi$ contribution. The information obtained from the polarization analysis of the diffraction peaks can be very rich.

\subsection{Stokes parameters}

There is another way to characterizing the polarization. It is the use of the Stokes parameters. They are used in many contexts: in astrophysics, in the instrumentation to 
validate the quality of the beam lines and in x-ray physics to describe with common tools the effect of the interaction with matter. The Stokes parameters describe completely the polarization state of an electromagnetic radiation. It is a four component vector $\mathbf{S}$ such that:

$$
\mathbf{S}=\left(\begin{array}{c}
S_{0} \\
S_{1} \\
S_{2} \\
S_{3}
\end{array}\right)=\left(\begin{array}{c}
\left|E_{x}\right|^{2}+\left|E_{y}\right|^{2} \\
\left|E_{x}\right|^{2}-\left|E_{y}\right|^{2} \\
2 \mathfrak{R}\left(E_{x} E_{y}^{*}\right) \\
2 \mathfrak{I}\left(E_{x} E_{y}^{*}\right)
\end{array}\right)
$$

The polarization itself (or rate of polarization) is thus given by :

$$
p=\frac{\sqrt{S_{1}^{2}+S_{2}^{2}+S_{3}^{2}}}{S_{0}} .
$$

For practical purposes, one normalizes by $S_{0}$ and thus one gets for example:

- $(1,0,0,0)$ : unpolarized,

- $(1,1,0,0)$ : linearly polarized along $\mathrm{x}$,

- $(1,-1,0,0)$ : linearly polarized along $\mathrm{y}$,

- $(1,0,1,0)$ : linearly polarized at 45 degrees,

- $(1,0,0,1)$ : left-hand circularly polarized,

- $(1,0,0,-1)$ : right-hand circularly polarized.

\subsection{Quantization of the electromagnetic field}

In the next section we will give the expression of the interaction between the electromagnetic wave and an electron in an atom. For this purpose we have first to derive the quantization of the electromagnetic field. When it is confined in a volume $V$, the potential vector, $\mathbf{A}(\mathbf{r}, t)$, is expressed using the previous expressions and the annihilation $a_{\varepsilon, \mathbf{k}}$ and creation $a_{\varepsilon, \mathbf{k}}^{+}$operators:

$$
\mathbf{A}(\mathbf{r}, t)=\sum_{\varepsilon, \mathbf{k}} \mathbf{A}_{0, \mathbf{k}}\left(a_{\varepsilon, \mathbf{k}} e^{i(\mathbf{k} \cdot \mathbf{r}-\omega t)} \varepsilon+a_{\varepsilon, \mathbf{k}}^{+} e^{-i(\mathbf{k} \cdot \mathbf{r}-\omega t)} \varepsilon^{*}\right),
$$

with:

$$
\mathbf{A}_{0, \mathbf{k}}=\sqrt{\frac{\hbar}{2 \varepsilon_{0} V \omega_{k}}}
$$

The Hamiltonian of the field is given by :

$$
H_{R}=\sum_{\varepsilon, \mathbf{k}} \hbar \omega_{k}\left(a_{\varepsilon, \mathbf{k}}^{+} a_{\varepsilon, \mathbf{k}}+\frac{1}{2}\right) .
$$




\section{Process of interaction of light with an electron in an atom}

Having defining the electromagnetic waves, one can now consider its interaction with matter. In this section we consider the interaction of a photon with one electron making a transition between two atomic levels. From the interaction Hamiltonian, we shall have, at the end of this chapter, the important processes involved in the absorption and resonant and non resonant scattering phenomena.

\subsection{Linear and non linear interactions}

A first distinction between the linear and non linear interactions should be carried out. When there is a single incoming or/and a single outgoing photon in the interaction, the process is said linear. With more photons one gets the nonlinear physics involved for example in frequency doubling. The subject, extensively studied specially in the infra-red - visible - ultra-violet regime, very rarely concerns x-ray physics. Indeed it needs huge radiation power, that is typically $1 \mathrm{MW} / \mathrm{mm}^{2}$ or in other words around $10^{24}$ photons per second. This can be reached by laser but not at synchrotron, even with the very high brilliance one gets now. In a close future, with the free electron laser which gives pulsed x-ray source with brilliance 1000 times stronger than the synchrotron, the radiation power will be probably sufficient to reach the nonlinear phenomena in the x-ray regime. For the moment it is not yet done and thus the following sections are limited to the linear response.

\subsection{Interaction Hamiltonian}

The non relativistic Hamiltonian of one photon and one particle of mass $m$ and charge $q$ is given by $[6,15]^{1}$ :

$$
H=\frac{1}{2 m}(\mathbf{P}-q \mathbf{A})^{2}+q V-g_{L} \frac{q}{2 m} \mathbf{S} \cdot \mathbf{B}+H_{R}
$$

where $g_{L}$ is the Landé factor (=2 in this case). $H_{R}$ is the Hamiltonian of the field already seen in 3.3. The relativistic Hamiltonian brings terms resulting in a spinorbit component which can be neglected in the X-ray regime. The non relativistic Hamiltonian can be split between its non interaction part $H_{0}$ and a perturbation $V_{I}$ :

$$
H_{0}=\frac{p^{2}}{2 m}+q V+H_{R},
$$

${ }^{1}$ The Hamiltonian is in SI unit. When expressed in CGS unit, there is a $1 / c$ extra factor in front of A. 


$$
V_{I}=-\frac{q}{m} \mathbf{P} \cdot \mathbf{A}+\frac{q^{2}}{2 m} \mathbf{A}^{2}-g_{L} \frac{q}{2 m} \mathbf{S} \cdot \mathbf{B} .
$$

We consider a transition between an initial state and a final state. The initial state contains one photon with wave vector $\mathbf{k}_{i}$ and polarization $\varepsilon_{i}$ and one electron in the state $\varphi_{g}$. In the same way the final state contains the photon $\left(\mathbf{k}_{s}, \varepsilon_{s}\right)$ and the electron $\varphi_{f}$. The solutions of $H_{0}$ can be written:

$$
\begin{aligned}
\left|\phi_{g}\right\rangle & =\left|\varphi_{g} ; \mathbf{k}_{i}, \varepsilon_{i}\right\rangle, H_{0}\left|\phi_{g}\right\rangle=\mathscr{E}_{g}\left|\phi_{g}\right\rangle \quad \text { with } \mathscr{E}_{g}=E_{g}+\hbar \omega_{i}, \\
\left|\phi_{f}\right\rangle & =\left|\varphi_{f} ; \mathbf{k}_{s}, \varepsilon_{s}\right\rangle, H_{0}\left|\phi_{f}\right\rangle=\mathscr{E}_{f}\left|\phi_{f}\right\rangle \text { with } \mathscr{E}_{f}=E_{f}+\hbar \omega_{s},
\end{aligned}
$$

where $\mathscr{E}_{g}\left(\mathscr{E}_{f}\right)$ is the total initial (final) energy, $E_{g}\left(E_{f}\right)$ is the electron initial (final) energy and $\hbar \omega_{i},\left(\hbar \omega_{s}\right)$ the initial (final) photon energy. For practical purposes, we shall use this formulation in the context of transition from one electronic localized state state up (or down) to some unlocalized state. We shall thus ascribe a density of states $\rho_{f}$ to the final state which is supposed to be not zero on some energy range. In this context the transition probability from $\left|\phi_{g}\right\rangle$ to $\left|\phi_{f}\right\rangle$ is given by:

$$
W_{f g}=\frac{2 \pi}{\hbar}\left|\left\langle\phi_{f}\left|T_{I}\right| \phi_{g}\right\rangle\right|^{2} \rho_{f},
$$

where $T_{I}$ is the transition operator given by:

$$
T_{I}=V_{I}+V_{I} G\left(\mathscr{E}_{g}\right) V_{I}
$$

$G(\mathscr{E} g)$ is the resolvent (Green function) of the total Hamiltonian, that is :

$$
G\left(\mathscr{E}_{g}\right)=\lim _{\eta \rightarrow 0^{+}} \frac{1}{\mathscr{E}_{g}-H+i \eta} .
$$

The zero order approximation using $T_{I} \approx V_{I}$ gives the second golden rule. In the following we shall use the first order approximation where we replace the Green function of the total Hamiltonian by that of the ground state Hamiltonian. The transition probability we get is the first golden rule where:

$$
T_{I} \approx V_{I}+V_{I} G_{0}\left(\mathscr{E}_{g}\right) V_{I}
$$

Inserting in eq. 22 the expression of $V_{I}$ given in eq. 16 and using $q=-e, g_{L}=2$ and $\mathbf{B}=-i \mathbf{k} \times \mathbf{A}$, one gets to the second order in an $e / m$ expansion:

$$
\begin{aligned}
T_{I} & \approx \frac{e}{m}(\mathbf{P} \cdot \mathbf{A}-i \mathbf{S} \cdot \mathbf{k} \times \mathbf{A}) \\
& +\left(\frac{e}{m}\right)^{2}\left[\frac{m}{2} \mathbf{A} \cdot \mathbf{A}+(\mathbf{P} \cdot \mathbf{A}-i \mathbf{S} \cdot \mathbf{k} \times \mathbf{A}) G_{0}\left(\mathscr{E}_{g}\right)(\mathbf{P} \cdot \mathbf{A}-i \mathbf{S} \cdot \mathbf{k} \times \mathbf{A})\right]+O\left(\frac{e^{3}}{m^{3}}\right)
\end{aligned}
$$

In this expression, the terms containing just one $\mathbf{A}$ reveal process with one photon, that is absorption or emission. The terms with two A reveal situation with two pho- 
tons, one photon in and one photon out, that is a scattering process. Let's introduce the expression of the potential vector given in eq. 11 .

\subsection{Absorption and Emission}

When keeping the annihilation term alone, one gets the operator corresponding to the absorption process:

$$
T_{I, a b s}=\frac{e}{m} \sum_{\varepsilon, \mathbf{k}} A_{0, \mathbf{k}} a_{\varepsilon, \mathbf{k}}(\varepsilon \cdot \mathbf{P}-i \mathbf{S k} \times \varepsilon) e^{i(\mathbf{k} \cdot \mathbf{r}-\omega t)} .
$$

To get the absorption cross-section one introduces this operator in the expression 19 and we divide by the incoming flux $(c / V)$. We get:

$$
\begin{aligned}
\sigma_{a b s} & =\frac{V}{c} \sum_{f} \frac{2 \pi}{\hbar}\left|\left\langle\phi_{f}\left|T_{I, a b s}\right| \phi_{g}\right\rangle\right|^{2} \rho_{f} \\
& =\frac{4 \pi^{2} \alpha \hbar}{m^{2} \omega} \sum_{f}\left|\left\langle\varphi_{f}|\hat{O}| \varphi_{g}\right\rangle\right|^{2} \rho_{f}\left(E_{f}=E_{g}+\hbar \omega\right),
\end{aligned}
$$

where $\alpha=e^{2} /\left(2 \varepsilon_{0} h c\right) \cong 1 / 127.036$ is the fine structure constant, and the operator $\hat{O}$ is given by :

$$
\hat{O}=(\varepsilon \cdot \mathbf{P}-i \mathbf{S k} \times \varepsilon) e^{i \mathbf{k} \cdot \mathbf{r}} .
$$

The total energies are given by :

$$
\mathscr{E}_{g}=E_{g}+\hbar \omega, \quad \mathscr{E}_{f}=E_{f} .
$$

Most often the term corresponding to the magnetic field can be neglected in the $\mathrm{X}$-ray energy range. This means that we use $\hat{O} \cong \varepsilon \cdot \mathbf{P} e^{i \mathbf{k} \cdot \mathbf{r}}$.

The emission is treated identically by keeping the creation operator only. There is no incident flux and the normalization depends on the peculiar process. For a coupling between the continuum and a discrete final level, one gets an emission cross-section proportional to the absorption cross-section but with the total energies given by:

$$
\mathscr{E}_{f}=E_{f}+\hbar \omega, \quad \mathscr{E}_{g}=E_{g}
$$

\subsection{Scattering}

Now one considers the two photon case. For a scattering process, to get the intensity, one has to divide by the incoming flux $(c / V)$ and to multiply by the density of pho- 
ton in the final state $V\left(\hbar \omega_{s}\right)^{2} /(2 \pi \hbar c)^{3}$. One thus multiplies and divides the matrix element $\left\langle\phi_{f}|T| \phi_{n}\right\rangle$ by the square root of the previous factors. To get the scattering amplitude in the conventional unit scale, that is in number of electron, one also divides by the electron radius $r_{0}=e^{2} /\left(4 \pi \varepsilon_{0} m c^{2}\right)=2.82 \times 10^{-5} \AA$. We have thus to calculate:

$$
f=\frac{V \hbar \omega_{s}}{(2 \pi \hbar c)^{3 / 2} r_{0}}\left\langle\phi_{f}|T| \phi_{g}\right\rangle \text {. }
$$

\subsubsection{Thomson scattering}

We consider the first term with $\mathbf{A} \cdot \mathbf{A}$ in the equation 24 . The corresponding amplitude gives:

$$
\begin{aligned}
f & =\frac{V \hbar \omega_{s}}{(2 \pi \hbar c)^{3 / 2} r_{0}} \frac{e^{2}}{2 m}\left\langle\phi_{f}|\mathbf{A} \cdot \mathbf{A}| \phi_{g}\right\rangle \\
& =\frac{\omega_{s}}{\omega_{i}} \varepsilon_{s}^{*} \varepsilon_{i}\left\langle\phi_{f}\left|e^{i\left(\mathbf{k}_{i}-\mathbf{k}_{s}\right) \cdot \mathbf{r}}\left(a_{i} a_{s}^{+}+a_{s}^{+} a_{i}\right)\right| \phi_{g}\right\rangle .
\end{aligned}
$$

In the elastic case one gets the Thomson scattering:

$$
f_{0}=\varepsilon_{s}^{*} \cdot \varepsilon_{i}\left\langle\varphi_{g}\left|e^{i\left(\mathbf{k}_{i}-\mathbf{k}_{s}\right) \cdot \mathbf{r}}\right| \varphi_{g}\right\rangle=\varepsilon_{s}^{*} \cdot \varepsilon_{i} \int|\varphi(\mathbf{r})|^{2} e^{-i \mathbf{Q} \cdot \mathbf{r}} d^{3} \mathbf{r}
$$

where $\mathbf{Q}=\mathbf{k}_{s}-\mathbf{k}_{i}$

We now compare this result with the classical demonstration. Thus, Thomson scattering is the elastic interaction with a free electron. The vibrating electron acts as a source and the ratio of the radiated field $E_{r}(r)$ over the incoming field $E_{i}$ is:

$$
\frac{E_{r}(r)}{E_{i}}=-r_{0} \frac{e^{i k r}}{r} \cos \psi,
$$

$\psi$ being the angle between the incoming polarization and the outgoing one. It is the scattering angle only when the polarizations are parallel with the scattering plane. One has $\cos \psi=\varepsilon_{s}^{*} \cdot \varepsilon_{i}$. The scattering amplitude expressed in number of electron is then:

$$
f=\varepsilon_{s}^{*} \cdot \varepsilon_{i} .
$$

For one atom with a distribution of charge $\rho(r)$, one just has to integrate over it, taking into account the phase difference between the paths (Fig. 7). This one is given by:

$$
\Delta \phi(\mathbf{r})=\left(\mathbf{k}_{i}-\mathbf{k}_{s}\right) \cdot \mathbf{r}=-\mathbf{Q} \cdot \mathbf{r},
$$

and the scattering length is thus:

$$
f(\mathbf{Q})=\int \rho(\mathbf{r}) e^{-i \mathbf{Q} \cdot \mathbf{r}} d^{3} \mathbf{r},
$$


that in the limit $\mathbf{Q} \rightarrow 0$ yields $f(\mathbf{Q}=0)=Z$. Finally just multiplying by the scattering amplitude for one electron (eq. 35), one gets again the formula giving the Thomson scattering amplitude (eq. 33).

In periodic systems, the diffracted peaks are mainly due to this effect. The phase difference between the atoms of the unit cell is taken into account by the Bragg factor, $e^{i \mathbf{Q} \cdot \mathbf{R}_{a}}$, in such a way that the intensity is given by:

$$
I(\mathbf{Q}) \propto\left|\varepsilon_{s}^{*} \cdot \varepsilon_{i} \sum_{a} e^{i \mathbf{Q} \cdot \mathbf{R}_{a}} f_{0 a}(\mathbf{Q})\right|^{2},
$$

where $\mathbf{R}_{a}$ and $f_{0 a}$ are respectively the position and Thomson scattering amplitude of the atom $a$. The summation is over all the atoms of the unit cell. From this equation, one sees that there is no $\sigma-\pi$ Thomson scattering. Most often the electronic density can be considered as spherical around the atoms. Consequently the Thomson scattering for a specific diffraction vector $\mathbf{Q}$ is mostly isotropic. Finally note that a temperature dependent Debye-Waller factor must also be included in the Bragg factor to take into account the thermal disorder.

Fig. 7 Thomson scattering where is shown the incoming beam with wave vector $\mathbf{k}_{i}$ scattered at the center and at a point $\mathbf{r}$ in the atom giving thus a phase difference.

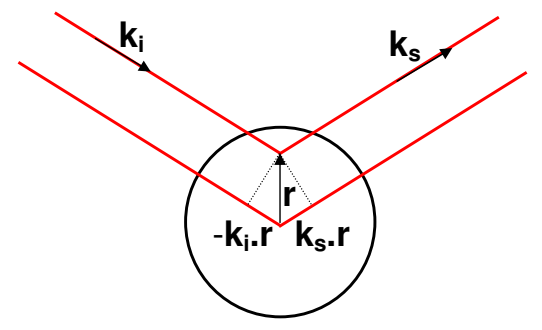

\subsubsection{Compton scattering}

For completeness one has to touch on the Compton scattering. It corresponds to the inelastic interaction with a free electron. Contrary to Thomson scattering, it cannot be explained purely with light as a wave phenomenon. One has to apply the relativistic energy and momentum conservations (Fig. 8), and we gets then:

$$
\frac{k_{i}}{k_{s}}=\frac{\hbar \omega_{i}}{\hbar \omega_{s}}=\frac{\lambda_{s}}{\lambda_{i}}=1+\lambda_{C} k_{i}(1-\cos \theta),
$$

where $\theta$ is the scattering angle and $\lambda_{C}=\hbar / m c$ is the Compton wavelength. the Compton scattering is incoherent and it is often seen as a simple background. This one is small in the x-ray range but increases at higher energy (see Fig. 8) and with 
the scattering angle. For electron in solid, it gives nevertheless information on the electronic momentum distribution (see chapter by S. P. Collins).

Fig. 8 Compton scattering with the ratio of the outgoing and incoming photon wave vectors versus the scattering angle and at different energies. The diagram in the inset shows the particles with their momenta.

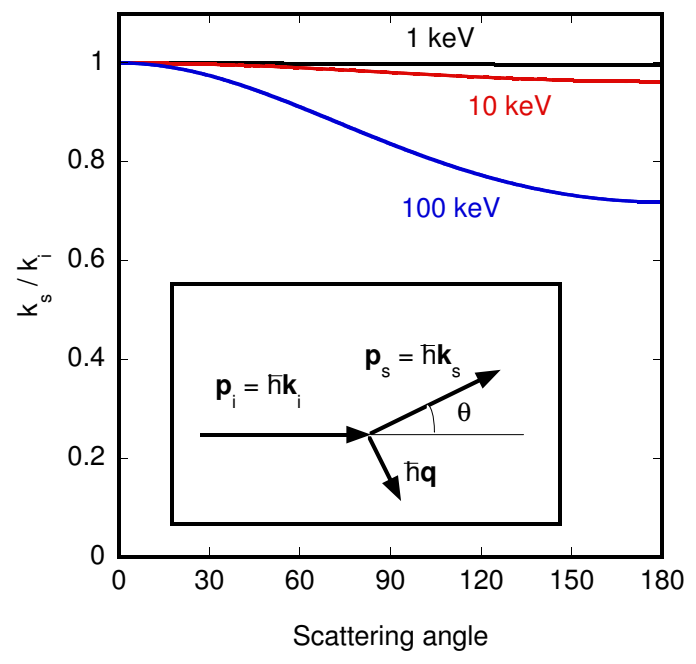

\subsubsection{Resonant scattering}

Now we consider the second term in the two photon process, the one containing $G_{0}\left(\mathscr{E}_{g}\right)$ in eq. 24 . This Green's function applied on the state $\left|\varphi_{n}\right\rangle$ is:

$$
G_{0}\left(\mathscr{E}_{g}\right)\left|\varphi_{n}\right\rangle=\lim _{\eta \rightarrow 0^{+}} \frac{\left|\varphi_{n}\right\rangle\left|\varepsilon_{n}, \mathbf{k}_{n}\right\rangle}{\mathscr{E}_{g}-H_{0}+i \eta}
$$

There are two possibilities. In the first one, the intermediate state already contains the photon of energy $\hbar \omega_{s}$. In other word, the emitted photon is created before the annihilation of the incoming one. We have thus:

$$
\mathscr{E}_{n}=E_{n}+\hbar \omega_{s}
$$

In the second case, there is no photon in the intermediate state. The emitted photon is created after the annihilation of the incoming one. In this case one gets:

$$
\mathscr{E}_{n}=E_{n} .
$$

In both cases one also has for the initial state $\mathscr{E}_{g}=E_{g}+\hbar \omega_{i}$. Consequently the expression of the scattering amplitude becomes:

Resonant case: 


$$
f_{(a)}=\frac{\omega_{s}}{\omega_{i}} \frac{1}{m} \lim _{\eta \rightarrow 0^{+}} \sum_{n} \frac{\left\langle\varphi_{f}\left|\hat{O}_{s}^{*}\right| \varphi_{n}\right\rangle\left\langle\varphi_{n}\left|\hat{O}_{i}\right| \varphi_{g}\right\rangle}{E_{g}-E_{n}+\hbar \omega_{i}+i \eta}
$$

Non resonant case with the photon in the intermediate state:

$$
f_{(b)}=\frac{\omega_{s}}{\omega_{i}} \frac{1}{m} \sum_{n} \frac{\left\langle\varphi_{f}\left|\hat{O}_{i}\right| \varphi_{n}\right\rangle\left\langle\varphi_{n}\left|\hat{O}_{s}^{*}\right| \varphi_{g}\right\rangle}{E_{g}-E_{n}-\hbar \omega_{s}}
$$

In these expressions the operator $\hat{O}_{i, s}$ has now subscripts corresponding to the incoming and outgoing photons and thus their corresponding wave vector and polarization. Note that for the expression of $f_{(b)}$ one does not need the limit with $\eta$ because $E_{g}-E_{n}$ and $\hbar \omega_{s}$ are generally of the same sign. On the contrary, $f_{(a)}$ is a resonant process because we can have $E_{g}-E_{n}+\hbar \omega_{s}=0$. We get a virtual transition because there is an uncertainty on the energy during the time $\Delta t$, such that $\Delta t \Delta E=\hbar$ with $\Delta E=\left|E_{n}-E_{g}-\hbar \omega_{s}\right|$. One can also remark that around an absorption edge the denominator of the resonant term is nearly zero. On the contrary the denominator of $f_{(b)}$ is around 2 times the energy edge. Thus $f_{(b)}$ is nearly always negligible in comparison with $f_{(a)}$.

The resonant term is used in many spectroscopies. Depending on the wave-length different properties of the material can be investigated. Section 6 is partly devoted to its use in the X-ray regime.

\subsubsection{Non-resonant magnetic scattering}

To end up this part on scattering, one can consider the non-resonant magnetic scattering. This scattering was explained by Blume and Doon Gibbs [7] and first observed in 1981 by de Bergevin and Brunel [17]. It contains the interaction between the magnetic field of the incoming wave with the spin of the electron. It contains also an angular momentum part. This one comes from the expansion at the first order in $\hbar \omega /\left(E_{n}-E_{g}\right)$ of the two resonant $f_{(a)}$ and non-resonant $f_{(b)}$ scattering amplitudes. At high energy one then gets a magnetic scattering amplitude given by:

$$
f_{m}=-\frac{\hbar \omega}{m c^{2}}\left(\frac{1}{2} \mathbf{L}(\mathbf{Q}) \cdot \mathbf{a}+\mathbf{S}(\mathbf{Q}) \cdot \mathbf{b}\right),
$$

where $\mathbf{a}$ and $\mathbf{b}$ are two vectors depending on the incoming and outgoing wave vectors and polarizations:

$$
\begin{aligned}
& \mathbf{a}=2\left(1-\mathbf{u}_{i} \cdot \mathbf{u}_{s}\right) \varepsilon_{s} \times \varepsilon_{i}-\left(\mathbf{u}_{i} \times \varepsilon_{i}\right) \mathbf{u}_{i} \cdot \varepsilon_{s}+\left(\mathbf{u}_{s} \times \varepsilon_{s}\right) \mathbf{u}_{s} \cdot \varepsilon_{i} \\
& \mathbf{b}=\varepsilon_{s} \times \varepsilon_{i}-\left(\mathbf{u}_{i} \times \varepsilon_{i}\right) \mathbf{u}_{i} \cdot \varepsilon_{s}+\left(\mathbf{u}_{s} \times \varepsilon_{s}\right) \mathbf{u}_{s} \cdot \varepsilon_{i}-\left(\mathbf{u}_{s} \times \varepsilon_{s}\right) \times\left(\mathbf{u}_{i} \times \varepsilon_{i}\right) .
\end{aligned}
$$

where $\mathbf{u}=\mathbf{k} / k . \mathbf{S}(\mathbf{Q})$ is the Fourier transform of the spin density, that is of $\rho(r)^{\uparrow}-$ $\rho(r)^{\downarrow} . \mathbf{L}(\mathbf{Q})$ is relied to the Fourier transform of the orbital momentum. It is often a good approximation to take $\mathbf{L}(\mathbf{Q})$ and $\mathbf{S}(\mathbf{Q})$ in the same direction. 
The amplitude of the non-resonant magnetic scattering is very small and neutron diffraction is most often far more sensitive to measure magnetic ordering. Nevertheless, when studying magnetic material using resonant processes, because this term interferes with the other terms, it can gives notable effects on the intensity of some peaks.

\subsection{Transition matrix}

In order to obtain the absorption and emission cross-sections and the resonant scattering amplitudes, matrix elements of the form $\left\langle\varphi_{f}|\hat{O}| \varphi_{g}\right\rangle$ have to be evaluated. The exponential in the operator can be expanded in terms of $\mathbf{k} \cdot \varepsilon$ and we gets at second order for respectively its electric and magnetic part:

$$
\begin{aligned}
& \hat{O}_{e}=\varepsilon \cdot \mathbf{P} e^{i \mathbf{k} \cdot \mathbf{r}}=\varepsilon \cdot \mathbf{P}\left(1+i \mathbf{k} \cdot \mathbf{r}-\frac{1}{2}(\mathbf{k} \cdot \mathbf{r})^{2}+\ldots\right), \\
& \hat{O}_{m}=-i \mathbf{S k} \times \varepsilon e^{i \mathbf{k} \cdot \mathbf{r}}=-i \mathbf{S k} \times \varepsilon\left(1+i \mathbf{k} \cdot \mathbf{r}-\frac{1}{2}(\mathbf{k} \cdot \mathbf{r})^{2}+\ldots\right) .
\end{aligned}
$$

For the first expression, the first term of the expansion is called the electric dipole or E1. The corresponding operator $\varepsilon \cdot \mathbf{P}$ can be rewritten thanks to its relation with the commutator:

$$
\varepsilon \cdot \mathbf{P}=\frac{m}{i \hbar}\left[\varepsilon \cdot \mathbf{r}, H_{0}\right] .
$$

Using the relation:

$$
\left\langle\varphi_{f}\left|\left[\varepsilon \cdot \mathbf{r}, H_{0}\right]\right| \varphi_{g}\right\rangle=\left(E_{g}-E_{f}\right)\left\langle\varphi_{f}|\varepsilon \cdot \mathbf{r}| \varphi_{g}\right\rangle,
$$

one gets:

$$
M_{f g, E 1}=\left\langle\varphi_{f}|\varepsilon \cdot \mathbf{P}| \varphi_{g}\right\rangle=i \frac{m}{\hbar}\left(E_{f}-E_{g}\right)\left\langle\varphi_{f}|\varepsilon \cdot \mathbf{r}| \varphi_{g}\right\rangle,
$$

Let us calculate now the second term of the expansion of the electric operator. To get the corresponding matrix one sets the polarization along the $y$-axis and the wave vector along $z$. Then we use:

$$
\begin{aligned}
\frac{m}{2 \hbar}\left[z y, H_{0}\right] & =\frac{m}{2 \hbar}\left(\left[z, H_{0}\right] y+z\left[y, H_{0}\right]\right)=\frac{i}{2}\left(P_{z} y+z P_{y}\right) \\
& =\frac{i}{2}\left(2 P_{z} y-P_{z} y+z P_{y}\right)=i P_{z} y-\frac{i}{2} L_{x},
\end{aligned}
$$

where $L_{x}$ is the $x$ component of the angular moment $\mathbf{L}$. For any direction we thus get:

$$
i \mathbf{P} \cdot \varepsilon \mathbf{k} \cdot \mathbf{r}=\frac{m}{2 \hbar}\left[\varepsilon \cdot \mathbf{r k} \cdot \mathbf{r}, H_{0}\right]+\frac{i}{2} \mathbf{k} \times \varepsilon \cdot \mathbf{L} .
$$


The first term of the second member of this expression gives the E2 contribution, or electric quadrupole:

$$
M_{f g, E 2}=i \frac{m}{\hbar}\left(E_{f}-E_{g}\right) \frac{i}{2}\left\langle\varphi_{f}|\varepsilon \cdot \mathbf{r k} \cdot \mathbf{r}| \varphi_{g}\right\rangle
$$

The second term of the second member depending on orbital moment $\mathbf{L}$ is magnetic. Consequently it is grouped with the zero order of the expansion coming from the spin contribution. The new term is called the magnetic dipole or M1:

$$
M_{f g, M 1}=\frac{1}{2}\left\langle\varphi_{f}|\mathbf{k} \times \varepsilon \cdot(\mathbf{L}+2 \mathbf{S})| \varphi_{g}\right\rangle .
$$

The M1 contribution is very small in the x-ray energy range because the radial integral part in its calculation is nearly zero (its selection rules gives $\Delta \ell=0$, it acts only on $m$ and $\sigma$ ).

\subsection{Selection rules}

We have to calculate:

$$
\left\langle\varphi_{f}|\hat{o}| \varphi_{g}\right\rangle=\sum_{\sigma} \int \varphi_{f}^{*}(\mathbf{r}, \sigma) \hat{o}(\mathbf{r}) \varphi_{g}(\mathbf{r}, \sigma) d^{3} \mathbf{r}
$$

When considering only the E1 and E2 transitions, there is no possibility of spin-flip during the transition, thus the sum on the spin is reduced to one index, $\sigma$, set outside. Here the operator just contains the terms inside the matrix of eq. 52 for E1 and eq. 55 for E2, that is:

$$
\hat{o}=\varepsilon \cdot \mathbf{r}\left(1+\frac{1}{2} i \mathbf{k} \cdot \mathbf{r}\right) .
$$

The core state $g$ is localized, thus the integral has to be performed only inside the absorbing atom. The expansion of $f, \hat{o}$ and $g$ in spherical harmonics is consequently very convenient because it singles out the angular momentum quantum numbers explicitly, and separates the radial and angular dependences.

\subsubsection{Final states}

In the non magnetic case, the final (or intermediate) state can be written as:

$$
\varphi_{f}(\mathbf{r})=\sum_{\ell, m} a_{\ell, m}^{f} b_{\ell}(E, r) Y_{\ell}^{m}(\Omega),
$$

where $\mathbf{r}=(r, \Omega)$ is expressed in spherical coordinates, $b_{\ell}(E, r)$ is the radial component of the wave function inside the atom. It is obtained by solving the radial 
Schrödinger equation in the atom. It weakly depends on the photo electron energy $E$. The $a_{\ell, m}^{f}$ are the amplitudes ${ }^{2}$.

In the magnetic case, if one neglects the spin-orbit, a single sum over $\sigma$ is to be included:

$$
\varphi_{f}(\mathbf{r})=\sum_{\ell, m, \sigma} a_{\ell, m, \sigma}^{f} b_{\ell, \sigma}(E, r) Y_{\ell}^{m}(\Omega) \chi_{\sigma}
$$

where the spin state is given by:

$$
\chi_{\frac{1}{2}}=\left(\begin{array}{l}
1 \\
0
\end{array}\right), \chi_{-\frac{1}{2}}=\left(\begin{array}{l}
0 \\
1
\end{array}\right)
$$

When one considers the spin-orbit, the final state is written as:

$$
\varphi_{f}(\mathbf{r})=\sum_{\ell, m, s} a_{\ell, m+\frac{1}{2}-s, s}^{f} b_{\ell, m+\frac{1}{2}-\sigma, s}^{\sigma}(E, r) Y_{\ell}^{m+\frac{1}{2}-\sigma}(\Omega) \chi_{\sigma}
$$

Note that in this case, the spherical harmonics are necessarily the complex ones. This expression comes from the solution in the atom of the Dirac equation. $\sigma$ is not anymore a good quantum number. We replace it by the index $s$. Due to the spin-orbit the $\left(\ell, m, \frac{1}{2}\right)$ and $\left(\ell, m+1,-\frac{1}{2}\right)$ components are part of the same state. This is what gives the eventual spin-flip during the photo electron scattering.

\subsubsection{Initial states}

Now let us look at the initial states. They are localized and the expansion seen in the previous expression is limited to one or two components. For example at the K-edge, one has $\ell=0$ and two initial states:

$$
\begin{aligned}
\left|\frac{1}{2},-\frac{1}{2}\right\rangle & =b_{0 \frac{1}{2}}(r) Y_{0}^{0} \chi_{-\frac{1}{2}} \\
\left|\frac{1}{2}, \frac{1}{2}\right\rangle & =b_{0 \frac{1}{2}}(r) Y_{0}^{0} \chi_{\frac{1}{2}} .
\end{aligned}
$$

At the $\mathrm{L}_{I I}$-edge; one has $\ell=1, j=1 / 2$ and two initial states:

$$
\left|\frac{1}{2},-\frac{1}{2}\right\rangle=b_{1 \frac{1}{2}}(r)\left(-\sqrt{\frac{2}{3}} Y_{1}^{-1} \chi_{\frac{1}{2}}+\sqrt{\frac{1}{3}} Y_{1}^{0} \chi_{-\frac{1}{2}}\right),
$$

\footnotetext{
${ }^{2}$ When using the monoelectronic approach, the $a_{\ell, m}^{f}$ contain the main dependence on the energy $E$. They are obtained using the continuity of the wave function and its derivative between the atom and its surrounding (see Sect.6.4 and 7.2). In the other approaches, they are just normalization coefficients, and the dependence in function of energy is put outside of the transition matrix. At this stage, whatever is the method, our demonstration is general.
} 


$$
\left|\frac{1}{2}, \frac{1}{2}\right\rangle=b_{1 \frac{1}{2}}(r)\left(-\sqrt{\frac{1}{3}} Y_{1}^{0} \chi_{\frac{1}{2}}+\sqrt{\frac{2}{3}} Y_{1}^{1} \chi_{-\frac{1}{2}}\right) .
$$

At the $\mathrm{L}_{I I I}$-edge; one has $\ell=1, j=3 / 2$ and four initial states:

$$
\begin{aligned}
\left|\frac{3}{2},-\frac{3}{2}\right\rangle & =b_{1 \frac{3}{2}}(r) Y_{1}^{-1} \chi_{-\frac{1}{2}}, \\
\left|\frac{3}{2},-\frac{1}{2}\right\rangle & =b_{1 \frac{3}{2}}(r)\left(\sqrt{\frac{1}{3}} Y_{1}^{-1} \chi_{\frac{1}{2}}+\sqrt{\frac{2}{3}} Y_{1}^{0} \chi_{-\frac{1}{2}}\right), \\
\left|\frac{3}{2}, \frac{1}{2}\right\rangle & =b_{1 \frac{3}{2}}(r)\left(\sqrt{\frac{2}{3}} Y_{1}^{0} \chi_{\frac{1}{2}}+\sqrt{\frac{1}{3}} Y_{1}^{1} \chi_{-\frac{1}{2}}\right), \\
\left|\frac{3}{2}, \frac{3}{2}\right\rangle & =b_{1 \frac{3}{2}}(r) Y_{1}^{1} \chi_{\frac{1}{2}} .
\end{aligned}
$$

In general when using complex harmonics one can just write:

$$
\varphi_{g}=\sum_{\sigma} G_{g}^{\sigma} b_{g}(r) Y_{\ell_{g}}^{m_{g}+\frac{1}{2}-\sigma} \chi_{\sigma} .
$$

\subsubsection{Operator}

The operator can also be expanded in spherical harmonics:

$$
\hat{o}=\varepsilon \cdot \mathbf{r}\left(1+\frac{1}{2} i \mathbf{k} \cdot \mathbf{r}\right)=\sum_{\ell_{o}, m_{o}}\left(\frac{i}{2} k\right)^{\ell_{o}-1} c_{\ell_{o}, m_{o}} r^{\ell_{o}} Y_{\ell_{o}}^{m_{o}}(\Omega)
$$

where the $c_{\ell_{o}, m_{o}}$ are specific coefficients with their operator quantum numbers $\left(\ell_{o}, m_{o}\right)$. For example for a polarization along $z$ and a wave vector along $x$ one gets:

$$
\begin{gathered}
\varepsilon \cdot \mathbf{r}=z=r \cos \theta=\sqrt{\frac{4 \pi}{3}} r Y_{1}^{0}, \\
\frac{i}{2} \varepsilon \cdot \mathbf{r k} \cdot \mathbf{r}=\frac{i}{2} k z x=\frac{i}{2} k r^{2} \sin \theta \cos \varphi=\frac{i}{2} k \sqrt{\frac{4 \pi}{15}} r^{2} Y_{2}^{1},
\end{gathered}
$$

where we have used the real spherical harmonics. These ones can eventually be expressed in terms of the complex spherical harmonics.

\subsubsection{The transition matrix}

We can now gather the equations 62,67 and 68 . The transition matrix for each spin $\sigma$ is then: 


$$
\begin{aligned}
\left\langle\varphi_{f}|\hat{o}| \varphi_{g}\right\rangle_{\sigma} & =\sum_{o}\left(\frac{i}{2} k\right)^{\ell_{o}-1} c_{\ell_{o}, m_{o}} \sum_{\ell, m} \Gamma_{\ell, m+\frac{1}{2}-\sigma}^{\ell_{g}, m_{g}+\frac{1}{2}-\sigma, \ell_{o}, m_{o}} \\
& \times \sum_{s} \mathscr{R}_{\ell, m+\frac{1}{2}-\sigma, s}^{g, \ell_{o}, \sigma}(E) a_{\ell, m+\frac{1}{2}-s, s}^{f},
\end{aligned}
$$

where:

$$
\mathscr{R}_{\ell, m+\frac{1}{2}-\sigma, s}^{g, \ell_{0}, \sigma}(E)=\int_{0}^{R} b_{\ell, m+\frac{1}{2}-s, s}^{\sigma *}(E, r) b_{g}(r) r^{2+\ell_{o}} d r,
$$

is the radial integral performed up to the atom radius $R$, and

$$
\Gamma_{\ell, m}^{\ell_{g}, m_{g}, \ell_{o}, m_{o}}=G_{g}^{\sigma} \int_{\text {sphere }} Y_{\ell}^{m *}(\Omega) Y_{\ell_{o}}^{m_{o}}(\Omega) Y_{\ell_{g}}^{m_{g}}(\Omega) d \Omega,
$$

is the angular integral or Gaunt coefficient multiplied by the factor $G_{g}^{\sigma}$ coming from the initial state. It is usually expressed in terms of Clebsch-Gordon coefficients. The angular integral is not zero only for peculiar value of $\ell, m$ :

- $\ell$ must have the same parity than $\ell_{g}+\ell_{o}$,

- $\left|\ell_{g}-\ell_{o}\right| \leq \ell \leq\left|\ell_{g}+\ell_{o}\right|$,

- $m=m_{o}+m_{g}$.

The last condition on $m$ is when using complex spherical harmonics. When using the real ones the conditions are:

- when $m_{o} m_{g}=0, m=m_{o}+m_{g}$,

- when $m_{o} m_{g}>0, m=\left|m_{o}+m_{g}\right|$ and $m=\left|m_{o}-m_{g}\right|$,

- when $m_{o} m_{g}<0, m=-\left|m_{o}\right|-\left|m_{g}\right|$ and $m=-\left|m_{o}+m_{g}\right|$.

For the dipole and quadrupole components, we have respectively $\ell_{o}=1$ and $\ell_{o}=2$. Thus, the difference on $\ell$ between the initial and the final state is:

- dipole: $\Delta \ell= \pm 1$,

- quadrupole: $\Delta \ell=0, \pm 2$.

The orbitals probed at the different edges are summarized in Table 1.

Table 1 Probed states for the dipole and quadrupole transition for the different edges

\begin{tabular}{lll}
\hline Edge & Dipole probed state & Quadrupole probed state \\
\hline $\mathrm{K}, \mathrm{L}_{I}, \mathrm{M}_{I}, \mathrm{~N}_{I}, \mathrm{O}_{I}$ & $\mathrm{p}$ & $\mathrm{s}-\mathrm{d}$ \\
$\mathrm{L}_{I I}, \mathrm{~L}_{I I I}, \mathrm{M}_{I I}, \mathrm{M}_{I I I}, \mathrm{~N}_{I I}, \mathrm{~N}_{I I I}, \mathrm{O}_{I I}, \mathrm{O}_{I I I}$ & $\mathrm{~s}-\mathrm{d}$ & $\mathrm{p}-\mathrm{f}$ \\
$\mathrm{M}_{I V}, \mathrm{M}_{V}, \mathrm{~N}_{I V}, \mathrm{~N}_{V}, \mathrm{O}_{I V}, \mathrm{O}_{V}$ & $\mathrm{p}-\mathrm{f}$ & $\mathrm{s}-\mathrm{d}-\mathrm{g}$ \\
\hline
\end{tabular}

At the K-edge, the initial state is $1 s$ and the Gaunt coefficients are equal to $\frac{1}{\sqrt{4 \pi}} \delta_{\ell, \ell_{o}} \delta_{m, m_{o}}$. The corresponding effect of the selection rule on the probed orbital is shown in Fig. 9. One considers a sample containing an octahedron surrounding, for instance, a $3 d$ element. This one is oriented in a specific way with respect to 
the incoming electromagnetic wave, which has its polarization along $z$ and the wave vector along $x$. The electric field of the wave makes that the core $1 s$ electron is vibrating at the first order (for the dipole) in the vertical direction, like a $p_{z}$. The core electron can thus jump into the empty $4 p_{z}$ state above the Fermi level. When the energy is sufficiently high, or the wave length sufficiently short in comparison with the $1 s$ orbital radius, the vibration is not anymore exactly along $z$. There is a phase difference along $x$ resulting in a $d_{x z}$-like vibration component. The core electron can thus jump into the $3 d_{x z}$ final state. This is a quadrupole transition.

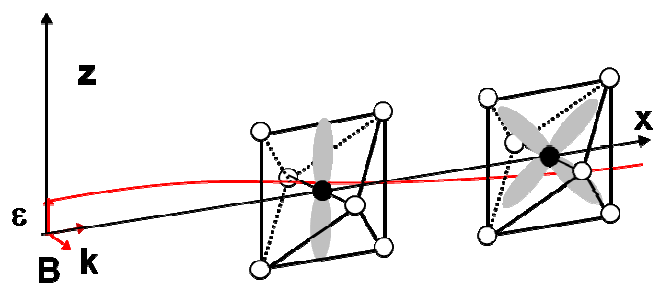

Fig. 9 Selection rule in the K-edge case. An octahedron is surrounding a $3 d$ element. When the polarization is along $z$, and the wave vector along $x$, the dipole probed orbital is the $p_{z}$ one, and the quadrupole probed orbital is the $3 d_{x z}$ one. When rotating the sample by 90 degrees, the probed $3 d$ state by the E2 channel can correspond to an $e_{g}$ state or to a $t_{2 g}$ state.

\section{Dielectric function or macroscopic point of view}

We briefly recall the definitions of the values permitting to describe macroscopically a dielectric. The aim is to make the connection of the susceptibility, permittivity and refractive index with the scattering factor used in the microscopic point of view.

A dielectric is a nonconducting substance where any volume element $d \tau$, have a dipole electric moment $d \mathscr{M}$. This one can be permanent or induced by an external electric fields $\mathbf{E}$. The polarization vector $\mathbf{P}$ is defined by:

$$
d \mathscr{M}=\mathbf{P} d \tau .
$$

The electric displacement at a point $M$ is defined by:

$$
\mathbf{D}(M)=\varepsilon_{0} \mathbf{E}(M)+\mathbf{P}(M) .
$$

For a linear (see Sect. 4.1) dielectric media the relation between the electric displacement and the electric field is given my: 


$$
\left(\begin{array}{c}
D_{x} \\
D_{y} \\
D_{z}
\end{array}\right)=\left(\begin{array}{ccc}
\varepsilon_{x x} & \varepsilon_{x y} & \varepsilon_{x z} \\
\varepsilon_{y x} & \varepsilon_{y y} & \varepsilon_{y z} \\
\varepsilon_{z x} & \varepsilon_{z y} & \varepsilon_{z z}
\end{array}\right)\left(\begin{array}{c}
E_{x} \\
E_{y} \\
E_{z}
\end{array}\right) .
$$

We define the electric susceptibility $\chi$ and the relative permittivity $\varepsilon_{r}$ with $\varepsilon=$ $\varepsilon_{0} \varepsilon_{r}=\varepsilon_{0}(1+\chi)$. Thus one gets:

$$
\mathbf{P}=\varepsilon_{0} \chi \mathbf{E}
$$

For an isotropic material, the refractive index is just given by:

$$
n=\sqrt{\varepsilon_{r}} .
$$

For an anisotropic material, the refractive index is a vector defined by:

$$
\mathbf{n}=\frac{c}{\omega} \mathbf{k} .
$$

where $\mathbf{k}$ is the wave vector, $c$ the speed of light and $\omega$ the pulsation. One gets the components of the refractive index from the Maxwell equations. Indeed for a plane wave we find:

$$
\mathbf{D}=\mathbf{n}^{2} \mathbf{E}-(\mathbf{n} \cdot \mathbf{E}) \mathbf{n} .
$$

Using the matrix relation between $\mathbf{D}$ and $\mathbf{E}$ and substituting $\mathbf{D}$ by eq. 80, one obtains the Fresnel equation:

$\mathbf{n}^{2}\left(\widehat{\varepsilon}_{x} n_{x}^{2}+\widehat{\varepsilon}_{y} n_{y}^{2}+\widehat{\varepsilon}_{z} n_{z}^{2}\right)-n_{x}^{2} \widehat{\varepsilon}_{x}\left(\widehat{\varepsilon}_{y}+\widehat{\varepsilon}_{z}\right)-n_{y}^{2} \widehat{\varepsilon}_{y}\left(\widehat{\varepsilon}_{x}+\widehat{\varepsilon}_{z}\right)-n_{z}^{2} \widehat{\varepsilon}_{z}\left(\widehat{\varepsilon}_{x}+\widehat{\varepsilon}_{y}\right)+\widehat{\varepsilon}_{x} \widehat{\varepsilon}_{y} \widehat{\varepsilon}_{z}=0$.

where $\left(\widehat{\mathcal{\varepsilon}}_{x}, \widehat{\mathcal{\varepsilon}}_{y}, \widehat{\mathcal{\varepsilon}}_{z}\right)$ are the eigenvalues of the permittivity matrix, defining the principal axis.

\section{Complex permittivity}

In this section we use classical equations to define a resonant system. When comparing with the quantum mechanical scattering factor calculated in Sect 4.4.3, we shall see that the resulting expressions have strong similarities. First we consider the differential equation of motion of an electron in a molecule:

$$
m \frac{d^{2} \mathbf{r}}{d t^{2}}=-k \mathbf{r}-\frac{m}{\tau} \frac{d \mathbf{r}}{d t}-e \mathbf{E}_{l},
$$

where $\mathbf{E}_{l}=\mathbf{E}+\mathbf{P} / 3 \varepsilon_{0}$ is the local field, $\tau$ some damping, $m, e$ and $\mathbf{r}$ the mass, absolute charge and position of the electron respectively. Putting $\mathbf{P}=2 \mathrm{Ner}$, where $N$ is the number of electron per unit volume, we find the relation between $\mathbf{E}$ and $\mathbf{P}$ and the electric susceptibility is given by: 


$$
\chi=\frac{\chi_{0} \omega_{1}^{2}}{\omega_{1}^{2}-\omega^{2}+i \frac{\omega}{\tau}}
$$

where:

$$
\chi_{0}=\frac{2 N e^{2}}{\varepsilon_{0} m \omega_{1}^{2}},
$$

is the susceptibility at $\omega=0$. From the equation 83 , the complex relative permittivity (or dielectric function) can be expressed as:

$$
\varepsilon_{r}(\omega)=\varepsilon_{r}^{\prime}(\omega)+i \varepsilon_{r}^{\prime \prime}(\omega)
$$

where its real and imaginary parts are given by:

$$
\varepsilon_{r}^{\prime}(\omega)-1=\frac{\chi_{0} \omega_{1}^{2}\left(\omega_{1}^{2}-\omega^{2}\right)}{\left(\omega_{1}^{2}-\omega^{2}\right)^{2}+\frac{\omega^{2}}{\tau^{2}}}, \quad \varepsilon_{r}^{\prime \prime}(\omega)=\frac{\chi_{0} \omega_{1}^{2} \frac{\omega}{\tau}}{\left(\omega_{1}^{2}-\omega^{2}\right)^{2}+\frac{\omega^{2}}{\tau^{2}}}
$$

When plotting (Fig. 10) these quantities, we find the typical resonant shapes for the imaginary and real part of the amplitude. From this simple consideration and taking into account that the main resonance pulsation $\omega_{1}$ are in the ultra-violet range, one gets the Cauchy formula giving the dependence of the refractive index on the wavelength at low frequency (that is in the visible):

$$
n^{2}=n_{0}^{2}+\frac{A}{\lambda^{2}}
$$

with $A=4 \pi^{2} \chi_{0} c^{2} / \omega_{1}^{2}$. We also obtain the value inferior to one for the refractive index at high frequency that is in the $\mathrm{x}$-ray range.

\section{Complex refractive index}

With a complex permittivity one also gets a complex refractive index and a complex wave vector. We have :

$$
k^{2}=\frac{\omega^{2}}{c^{2}}\left(\varepsilon_{r}^{\prime}+i \varepsilon_{r}^{\prime \prime}\right) \text {. }
$$

Setting $k=k^{\prime}+i k^{\prime \prime}$, one gets:

$$
k^{\prime 2}-k^{\prime \prime 2}=\frac{\omega^{2}}{c^{2}} \varepsilon_{r}^{\prime}, k^{\prime} k^{\prime \prime}=\frac{\omega^{2}}{c^{2}} \varepsilon_{r}^{\prime \prime} .
$$

So, for a plane wave along $z$, there is a damping given by $e^{i k z}=e^{i k^{\prime} z} e^{-k^{\prime \prime} z}$. The complex refractive index is simply defined by :

- $n^{\prime}=k^{\prime} c / \omega$ is the refractive index,

- $n^{\prime \prime}=k^{\prime \prime} c / \omega$ is the extinction index. 
Around $\omega_{1}$ there is an absorption and an abnormal dispersion. Far from $\omega_{1}$, there is no absorption and a normal dispersion.

From these relations, reflection and transmission coefficients can be calculated for an incident electromagnetic wave when interacting with a surface separating two media of different refractive indices. It is then possible to get a relation between the orientation of the polarization of an electromagnetic wave after reflection by a surface in term of the permittivity. In the visible range, one of the goals of ellipsometry is to find this parameter. Another one is, knowing the refractive index, to determine the thickness and roughness of a surface. For magnetic material, the equivalent technique is called Kerr effect.

At higher energy, in the soft x-ray range, reflectivity is a tool to get magnetic moment and thickness versus depth in a material. The connection with resonant scattering factor, $f(\mathbf{Q}, \omega)$, is in this technique effective and, we can write the relation between the refractive index $n$ and $f(\mathbf{Q}, \omega)$ :

$$
n(\mathbf{Q}, \omega)=1-\frac{2 \pi N_{a} r_{0}}{k^{2}} f(\mathbf{Q}, \omega)
$$

with $r_{0}$ the electron radius, $N_{a}$ the number of atom per volume unit and $\mathbf{Q}$ the diffraction vector. For a magnetic system the scattering factor itself depends, as seen previously, on a non resonant and a resonant part. The $\mathbf{Q}$ dependence of $n$ shows its angular dependence.

Fig. 10 Real and imaginary part of the permittivity for $\tau \omega_{1}=5$. At high frequency corresponding to the $\mathrm{x}$-ray range, the real part is negative. The refractive index is thus less than one.

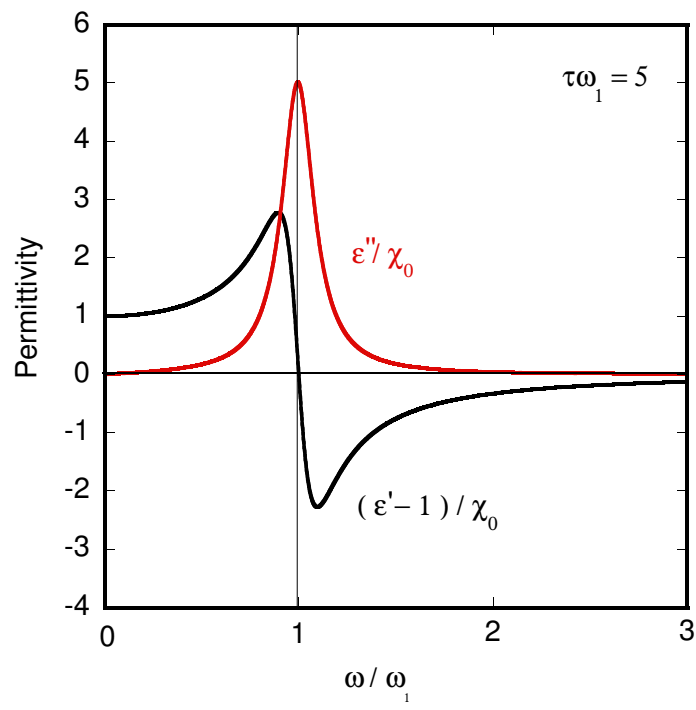




\section{X-ray spectroscopies}

In Sect. 4, we have seen the case of the interaction of a photon with an electron making a transition between two levels. It is implied that the electron is in some atom. In this section we focus now on the $\mathrm{x}$-ray spectroscopies. The corresponding energies make that the transition is necessarily between a core level and some other free level above the Fermi energy (or above the highest molecular occupied orbital to adopt the chemistry terminology).

The series of ionization edges follows the series of the atomic levels. They are thus labeled with the letter $\mathrm{K}, \mathrm{L}, \mathrm{M}, \mathrm{N}, \mathrm{O}$ according to the first atomic quantum number, $n=1,2,3,4$ and 5. The atomic levels are also split according to the second quantum number $\ell$ and to the third one $j=|\ell+s|$, where $s= \pm \frac{1}{2}$ is the spin. All these sub levels are labeled using the subscripts $I, I I, I I I, I V, V$ as is shown in Table 2.

Table 2 Correspondance between the edge names and the quantum numbers of the core states. The two last columns give the corresponding spectroscopic name and the number of electron.

\begin{tabular}{lllll}
\hline Edge & $\ell$ & $j$ & spectro & numb. of states \\
\hline $\mathrm{K}, \mathrm{L}_{I}, \mathrm{M}_{I}, \mathrm{~N}_{I}, \mathrm{O}_{I}$ & 0 & $1 / 2$ & $s_{1 / 2}$ & 2 \\
$\mathrm{~L}_{I I}, \mathrm{M}_{I I}, \mathrm{~N}_{I I}, \mathrm{O}_{I I}$ & 1 & $1 / 2$ & $p_{1 / 2}$ & 2 \\
$\mathrm{~L}_{I I}, \mathrm{M}_{I I I}, \mathrm{~N}_{I I I}, \mathrm{O}_{I I I}$ & 1 & $3 / 2$ & $p_{3 / 2}$ & 4 \\
$\mathrm{M}_{I V}, \mathrm{~N}_{I V}, \mathrm{O}_{I V}$ & 2 & $3 / 2$ & $d_{3 / 2}$ & 4 \\
$\mathrm{M}_{V}, \mathrm{~N}_{V}, \mathrm{O}_{V}$ & 2 & $5 / 2$ & $d_{5 / 2}$ & 6 \\
\hline
\end{tabular}

The expression of the core level wave functions corresponding to the $\mathrm{K}, \mathrm{L}_{I I}$ and $\mathrm{L}_{I I I}$ edges was given in Sect. 4.6.2. The energy of the edges follows the order of the atomic levels, $\mathrm{K}$ being the most energetic, then comes $\mathrm{L}_{I}, \mathrm{~L}_{I I}, \mathrm{~L}_{I I I}, \mathrm{M}_{I}, \mathrm{M}_{I I}$, $\mathrm{M}_{I I I}, \mathrm{M}_{I V}, \mathrm{M}_{V}$ and so on. They spread from $13.6 \mathrm{eV}$ for the K-edge of Hydrogen (defining the Rydberg unit) up to $115606 \mathrm{eV}$ for the K-edge of Uranium. The $\mathrm{K}$ edges of the light elements and the $\mathrm{L}_{I I, I I I}$ edges of the $3 d$ elements are in the soft $\mathrm{x}$-ray energy range.

In fact, the atom is embedded in a molecule or in a solid and most often, the arrival states for the photo electron cannot be considered as completely localized. The description is very different depending upon the localization of the arrival levels (atom-like or band-like). For the moment we remain with a final (or intermediate state) at least partially non localized and thus with some hybridization with the environment. The probability of transition depends on the transition matrix seen in Sect. 4 which itself depends on the final states. X-ray spectroscopies are thus necessarily a direct probe of the density of states, or at least its projection on the absorbing atom. This density of states depends on the position of the neighborhood atoms and thus absorption spectroscopies are also indirectly a probe of the geometrical surrounding (inter atomic distances and symmetry). 
The transition can be observed by different detection modes. One can simply measure the absorption rate, that is the damping of the x-ray beam through a sample. This is the most frequent measurement of $\mathrm{x}$-ray absorption fine structure spectra for samples in powder. For mono crystalline samples, or for surface sensitive measurements, fluorescence or secondary electron emission measurements, can be preferred. They are in principal nearly proportional to the transition process, but for practical purposes, strong divergence can be observed. One of the main reason comes from the self-absorption process.

Irrespective of the detection mode, X-ray absorption spectroscopies are local spectroscopies. They are selective on the chemical specie because of the position of the edge energy as well as on the second and third quantum numbers of the final states. However the processes involved are complex. There is no a single transition of one electron from one level to another level. The scheme is more complex because during this transition, all the other electrons would have to be included in the process. The photo electron probes final states which see a core-hole. When the interaction with the hole is too strong, it must be taken into account within a multielectronic scheme. The other electrons tend also to screen the core-hole. In any case, the probed density of states is not the ground density of states but an excited one.

\subsection{Characteristic times}

The main aspect in the interaction of light with matter, at least in the domain exposed here, is the absorption of a photon induced by a transition of a core (for the $\mathrm{x}$ ray energy) electron up to some higher level. The transition process of this single electron comes thus with a complete reorganization of the multi-electronic (and vibrating ...) configuration. The limit of validity of the mono electronic approach can be understood looking the different characteristic times in the absorption process:

- Time of process absorption of the photon. It is given by $t_{1}=1 / W_{f g}$ where $W_{f g}$ is the transition probability. We have $t_{1}<10^{-20} \mathrm{~s}$.

- Lifetime of the core-hole. It is given by $t_{2}=\hbar / \Delta E_{g}$ where $\Delta E_{g}$ is the core level width. For the $1 \mathrm{~s}$ level for $Z=20$ up tp $Z=30, \Delta E_{g} \approx 1 \mathrm{eV}$ and $t_{2}$ spreads from $10^{-15}$ down to $10^{-16} \mathrm{~s}$.

- Relaxation time of the electrons. It is the effect on all the electrons of the field created by the hole and the photo electron. There are many kinds of process. It is intrinsically multi-electronic. The associated time also ranges from $t_{3} \approx 10^{-15}$ down to to $10^{-16} \mathrm{~s}$.

- Transit time of the photo electron outward from the atom. It depends on the photo electron kinetic energy. For $E_{c}=1$ up to $100 \mathrm{eV}, t_{4}$ spreads from around $10^{-15}$ down to $10^{-17} \mathrm{~s}$.

- Thermal vibration. The time scale associated to the thermal displacements extends from about $10^{-11}$ down to $10^{-14} \mathrm{~s}$. 
We see that the thermal time scale is far larger that the other ones, thus in most cases the thermal aspect can be seen as independent of the other phenomena. For this adiabatic approximation, it is often said that the photon sees a rigid but slightly disordered arrangement of atoms. Nevertheless in the smooth $\mathrm{x}$-ray range, for example at the K-edge of carbon in small molecules, the orders of vibration can sometimes be observed. Nevertheless the usual energy range for this kind of observation remains the infra-red.

More interestingly, it can be noted that $t_{2}, t_{3}$ and $t_{4}$ can be of the same order. It is the condition to have multi-electronic processes involved. For this, the first condition is that the photo electron has a very low kinetic energy. The second condition, as stated above, is that the final state is localized. We know that it is typically the case of the $\mathrm{L}_{I I, I I I}$ edges of the $3 d$ elements and even more of the $\mathrm{M}_{I V, V}$ edges of the $4 f$ elements. Nevertheless, there is an experimental way to check when it is the case. One just has to look at the edge shape. When the edge is more or less step-like, the final states are not localized. It is the case of the K edges (but for some light elements when the electron probes bounded unoccupied molecular orbital), and at the $\mathrm{L}_{I I, I I I}$ edges of the heavy elements. On the contrary the edge shape can be more or less peak-like, that is that some $\mathrm{eV}$ after the usual increase, the absorption crosssection completely decreases, to go back to nearly zero. This means that the probed states are localized in energy as well as in space. In the first case the simulation of such spectra can be performed using mono-electronic simulations. The difficulties is that, the state being not localized, the electronic structure must be evaluated in a sufficiently large volume around the absorbing atom. In the second case, multielectronic calculations are necessary. When the localization is very high, atomic multi-configuration calculations where the surrounding symmetry breaking is just parametrized, is often sufficient. It is the domain of multiplet theory. Unfortunately it exists intermediate situations, where the edge starts with a high peak, the absorption cross-section then decreases rather strongly but does not completely vanish after the edge. In these cases, multi-electronic and multi-atomic calculations are mandatory. The theories that account for this multi-processes are actually in progress. There are three main tracks. The first uses a multi-channel approach [30], the second uses the Bethe-Salpeter equation [44] and the third the time-dependent density functional theory (TDDFT) [33, 2].

\subsection{The different spectroscopies}

We treat the spectroscopies related to the transition of a core level up to some excited level. We recall that this transition which induces a photon absorption can be real or virtual. 
Real absorption

$\mathrm{X}$-ray absorption spectroscopy (XAS) is the general name for the techniques measuring in some way the photon absorption. A distinction is first made according to the energy range of study. In the wide range from around $50 \mathrm{eV}$ after the edge up to several hundredth (or even some thousands) of eV, the spectroscopy is called EXAFS for extended x-ray absorption fine structure. This spectroscopy is specially sensitive to the radial distances of the different shells of neighboring atoms ( 2 shells, sometimes more) around the absorbing atom. It also gives the number of atoms by shell albeit with less precision, because this parameter is correlated with the thermal disorder. The data analysis is performed by comparison with simulations where the calculated spectra is built by simple superposition of outgoing and backscattered electron waves by the different shells. It is the interference phenomena on the absorbing atom which depends on the shell distances from the central atom, and on the photo electron energy which is responsible of the oscillating aspect of the spectra [32] and thus of the sensitivity of the method. Actual analyzes go farther than the single scattering process by considering a multiple scattering phenomena limited to some paths between the atoms.

At low kinetic energy of the photo electron, that is from the rising edge up to around $100 \mathrm{eV}$, the absorption spectroscopy is called XANES for X-ray absorption near edge spectroscopy. This spectroscopy is sensitive to the three-dimensional aspect of the geometry around the absorbing atom. As stated above, it is also directly sensitive to the electronic structure around the absorbing atom.

In the XANES energy range, analysis as a function of the polarization are often done. Using a single crystal of a media with some preferential arrangement, as on a surface, one can perform study with different orientations of the samples. When the incoming electromagnetic wave field is linearly polarized, the corresponding spectroscopy is called linear dichroism. When it is circularly polarized, one usually makes measurements under right and left polarizations and one computes the difference (Fig. 5). This technique is specially useful to study ferro or ferri magnetic systems (in antiferro it gives no intensity!). It is called $x$-ray magnetic circular dichroism (XMCD). Without magnetism this technique also exists for peculiar symmetry of material. It gives small but detectable signal. It is called natural circular dichroism. More recently a new technique has surged with the time analysis of the rotation of orbitals under magnetic field. It is called $x$-ray detected magnetic resonance (XDMR).

From the absorption techniques, one derives many other techniques as $x$-ray photo electron spectroscopy (XPS) or with angular resolution on the emitted electron photodiffraction. At lower energy and with a better resolution it is called angular resolved photo electron spectroscopy (ARPES)[9]. The latter is specially adapted to study tiny details in the band structure and is actually extensively used for the analysis of supra conducting materials.

It can be noted that an equivalent spectroscopy is performed in the electronic microscope. It is the electron energy loss spectroscopy (EELS) where the incoming photons are replaced by an incoming energetic electron beam. The measured spec- 
tra are very similar (but with a slightly less good resolution) to the ones obtained at synchrotron. The main dipolar contribution is equivalent. The smaller quadrupolar one obtained when increasing the electron scattering angle is different. Recent studies claim for Angstrom lateral resolution thanks to the Angstrom-size electron beam.

Virtual absorption

When the transition is virtual, or as is more usually said, for resonant scattering process, different spectroscopies are used. In the context of elastic scattering in diffraction mode, a distinction is done according to the energy range, as in the XAS case. The equivalent to EXAFS is called diffraction anomalous fine structure spectroscopy (DAFS). The diffraction peaks are then measured along a wide energy range. On the contrary, when measuring spectra just around the edge (as in XANES), the DAFS is called diffraction anomalous near edge structure (DANES) or resonant $x$-ray spectroscopy (RXS) or resonant $x$-ray diffraction (RXD). There is a third technique where the intensity of the reflections is recorded only at some points in energy around the edge. This is a way to increase the sensitivity of the diffraction technique on some specific atoms (corresponding to the energy edge). This technique is called multi-wavelength anomalous diffraction (MAD). It is often used in complex material and specially in biology to help with the structure resolution.

\subsection{Fluorescence and Auger}

Two spectroscopies are a direct consequence of the photoelectric effect. Due to the expulsion of the electron from its atomic level, a hole remains in the atom. The atom is thus excited and two main concurrent channels can put back the atom in a less excited state. Both channels result from the transition of a second electron down to the level let free (Fig. 11). The resulting energy from this new transition can be directly emitted through a photon of energy $E_{1}-E_{2}$ or through a third electron of the atom (at level $E_{3}$ ) keeping the kinetic energy $E_{1}-E_{2}-E_{3}$. Both effects give typical signature of the material by the energy of their appearance and sometimes by the shape of the resulting spectra. The first gives the x-ray emission spectroscopy (XPS) or UPS in the ultra-violet regime, the second, gives the Auger electron spectroscopy (AES). ${ }^{3}$ The relative effect of both phenomena depends on the atomic number of the element. Below $Z=32$, the Auger emission dominates. At higher value, it is the $\mathrm{X}$-ray emission which is dominating. Note also that due to the difference in the detectable particle, the electron having a far less mean free path, the Auger technique

${ }^{3}$ Note that the first core hole can in the same way be created by an incident energetic electron and not a photon. 
is mainly surface sensitive and indeed it is one of the most popular technique to check the purity of a surface.

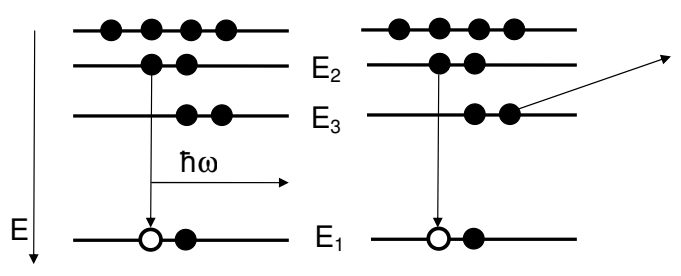

Fig. 11 The two channels for the desexcitation of an atom when a hole is left after the transition of a core electron. In the left panel, the transition of a secondary electron from level $\mathrm{E}_{2}$ down to the $E_{1}$ core level gives directly a photon of energy $E_{1}-E_{2}$. In the Auger effect, right panel, the corresponding energy is given to a third electron which keeps the kinetic energy $E_{1}-E_{2}-E_{3}$.

\subsection{XANES and RXS Formula}

The initial and final states must contain all the electrons of the system and one would have to calculate matrix terms of the form:

$$
\left\langle\Phi_{f}\left(r_{1}, r_{2}, r_{3}, \ldots\right)|\hat{o}| \Phi_{g}\left(r_{1}, r_{2}, r_{3}, \ldots\right)\right\rangle
$$

where $r_{1}, r_{2}, r_{3} \ldots$ are the positions of the different electrons and $\Phi$ the multielectronic states. Such a general formulation is developed in the Ligand Field Multiplet scheme. All the possible transitions from all the multi-electronic states up to all the multi-electronic final states are then sum up. They occur at different energies, each one with an amplitude related to the multiplicity of the states. The comb figure is then convoluted to get spectra to be compared with the experimental data. In the mono electronic approach we write $\Phi\left(r_{1}, r_{2}, r_{3}, \ldots\right)=\varphi_{1}\left(r_{1}\right) \varphi_{2}\left(r_{2}\right) \varphi_{3}\left(r_{3}\right) \ldots$ One also supposes that there is a specific electron which makes the transition, the others being spectators. The different states are seen through a continuum and with a density of states that can be calculated. In this condition the general formula seen in Sect. 4 still apply. The effect of the other electrons is then a simple multiplicative factor :

$$
S_{0}=\prod_{i=2, N}\left\langle\varphi_{i}^{f}\left(r_{i}\right) \mid \varphi_{i}^{g}\left(r_{i}\right)\right\rangle
$$

$S_{0}$ is typically of the order of 0.8 and is supposed to be energy independent. It comes from the fact that the core hole screening induces a contraction of the final state atomic orbitals resulting in the scalar product less than 1 . The screening also 
makes the energies of the other atomic levels change so that in the transition one has:

$$
\hbar \omega \cong \mathscr{E}_{f}-\mathscr{E}_{g}=E_{f}-E_{g}+\Delta E_{a},
$$

where $\Delta E_{a}$ is the sum of the differences between the initial and final energies of the other electrons. This fact is mostly often forgotten and $\Delta E_{a}$ is not written. Nevertheless it can represent several hundredth of $\mathrm{eV}$ in typical $\mathrm{K}$ edges.

With this simplification we can use the matrix product which appears in both absorption (eq. 26) and RXS (eq. 43) formulas:

$$
A=\sum_{f, g}\left\langle\varphi_{g}\left|\hat{o}_{s}^{*}\right| \varphi_{f}\right\rangle\left\langle\varphi_{f}\left|\hat{o}_{i}\right| \varphi_{g}\right\rangle .
$$

In the absorption cross-section case one just has to impose $\hat{o}_{s}=\hat{o}_{i}$. Using moreover the eq. 72 in the Sect. 4.6 on the selection rules which express the transition matrix in terms of the radial and angular integrals and of the atomic amplitudes, one gets:

$$
\begin{aligned}
A & =\sum_{o_{i}, o_{S}} c_{\ell_{o_{i}}, m_{o_{i}}} c_{\ell_{o_{s}}, m_{o_{S}}} \sum_{\sigma, g} \sum_{\ell, m, \ell^{\prime}, m^{\prime}} \Gamma_{\ell, m+\frac{1}{2}-\sigma}^{\ell_{g}, m_{g}+\frac{1}{2}-\sigma, \ell_{o_{i}}, m_{o_{i}}} \Gamma_{\ell, m^{\prime}+\frac{1}{2}-\sigma}^{\ell_{g}, m_{g}^{\prime}+\frac{1}{2}-\sigma, \ell_{o_{s}}, m_{o_{s}}} \\
& \times \sum_{s, s^{\prime}} \mathscr{R}_{\ell, m+\frac{1}{2}-\sigma, s}^{g, \ell_{o_{i}}, \sigma}(E) \mathscr{R}_{\ell, m+\frac{1}{2}-\sigma, s^{\prime}}^{g, \ell_{o_{s}}, \sigma}(E) \sum_{f} a_{\ell, m, s}^{f} a_{\ell^{\prime}, m^{\prime}, s^{\prime}}^{f *} .
\end{aligned}
$$

where we omit the index $f$ on the energy because in this summation, they all have the same value.

When using the monoelectronic approximation, the density of states does not appear explicitly in the formula because it is included in the atomic amplitudes and in the normalization of the radial wave functions. This one is built by continuity with an outer sphere where the potential is constant. The solutions are there, the Bessel and Hankel functions normalized by the density of states in vacuum, that is by $\sqrt{k / \pi}$ where $k$ is the photo-electron wave-vector. One thus gets the normalized radial solutions $\bar{b}_{\ell}(E, r)$ instead of the $b_{\ell}(E, r)$ which are normalized to one. In the same way one gets $\overline{\mathscr{R}}$ instead of $\mathscr{R}$. Thus one replaces in the formulas $\left\langle\varphi_{g}\left|\hat{o}_{s}^{*}\right| \varphi_{f}\right\rangle\left\langle\varphi_{f}\left|\hat{o}_{i}\right| \varphi_{g}\right\rangle \rho(E)$ by $\frac{1}{\pi}\left\langle\varphi_{g}\left|\hat{o}_{s}^{*}\right| \bar{\varphi}_{f}\right\rangle\left\langle\bar{\varphi}_{f}\left|\hat{o}_{i}\right| \varphi_{g}\right\rangle$.

In many cases this expression can be simplified. For example when quadrupole transitions are negligible as in the $\mathrm{L}_{I I, I I I}$ edges of $3 \mathrm{~d}$ elements, the summation on the operators often reduces to one element. In the absorption case where $\hat{o}_{s}=\hat{o}_{i}$ we can write:

$$
\begin{aligned}
\sigma & =4 \pi^{2} \alpha \hbar \omega c_{\ell_{o}, m_{o}}^{2} \sum_{\sigma, g} \sum_{\ell, m, s}\left(\Gamma_{\ell, m+\frac{1}{2}-\sigma}^{\ell_{g}, m_{g}+\frac{1}{2}-\sigma, \ell_{o}, m_{o}} \overline{\mathscr{R}}_{\ell, m+\frac{1}{2}-\sigma, s}^{g, \ell_{o_{i}}, \sigma}(E)\right)^{2} \\
& \times \frac{1}{\pi} \sum_{f}\left|a_{\ell, m, s}^{f}(E)\right|^{2}
\end{aligned}
$$


From this equation, one has to introduce a broadening due to the core-hole and final state life times to get a signal equivalent to the measured one. This is achieved by convoluting eq. 97 by a Lorentzian with an increasing width in energy. At the Fermi energy, it is typically the initial state width. At higher energy, plasmons and other phenomena contributes also to the damping. Often the width follows an arctangentlike function versus the photo electron kinetic energy. Note that new measurements improve the resolution by selecting sub-channel using the fluorescence detection. This limit the hole width to smaller value.

For the RXS, the infinitely small $\eta$ in eq. 43 is replaced by a finite value equivalent to the width broadening in absorption. The summation on the intermediate states $n$ can be made in two steps. We first group, as in XANES, the states of same energy (we shall call them now $f$ of energy $E$, to be homogeneous with the XANES formula), that we still sum in a discrete form. The sum over the states of different energies is performed in a second step through an integral spreading from the Fermi level up to infinity. In the elastic case where we are supposed to have $E-E_{g}+\Delta E_{a} \cong \hbar \omega$ the resonant or anomalous scattering amplitude is given by:

$$
f^{\prime}-i f^{\prime \prime} \cong m \omega^{2} \int_{E_{F}}^{\infty} \frac{\frac{1}{\pi} \sum_{f, g}\left\langle\varphi_{g}\left|\hat{o}_{s}^{*}\right| \bar{\varphi}_{f}\right\rangle\left\langle\bar{\varphi}_{f}\left|\hat{o}_{i}\right| \varphi_{g}\right\rangle}{\hbar \omega-\left(E-E_{g}+\Delta E_{a}\right)+i \frac{\Gamma}{2}} d E
$$

where $E_{F}$ is the Fermi energy and $\Gamma$ is the broadening. Note the minus sign in front of the imaginary part of the scattering amplitude to get the conventional way where $f^{\prime}$ is negative and $f^{\prime \prime}$ positive ${ }^{4}$. When the incoming and outgoing polarizations are parallel, the absorption cross-section is nearly proportional to $\hbar \omega \times f^{\prime \prime}$.

The RXS and XANES formula we have derived stand for an atom embedded in some surrounding. In fact the absorption or scattering comes from all the atoms in the molecule or in the unit cell. Thus one has to sum over them eventually using the symmetry operations of the space group. For the XANES this gives:

$$
\sigma_{\text {cell }}=\sum_{a=1}^{N} \sigma_{a}=\sum_{a=1}^{N_{e}} \sum_{b=1}^{n_{a}} S_{b}\left(\sigma_{a}\right)
$$

where $\mathrm{N}$ is the number of atom, $b$ is the index of the $n_{a}$ equivalent atoms related by the symmetry operation $S_{b}$ to the prototypical atom $a . N_{e}$ is the number of non equivalent atoms. We see in this way that the tensor dependence (or anisotropy) of the absorption cross-section depends not on the individual point group but on that of the unit cell. On the contrary, the shape of the spectra is related to the individual surroundings and symmetries.

For RXS, one uses the same summation but now taking into account the Bragg factors and the non resonant contributions. The intensity for a diffraction peak of diffraction wave vector $\mathbf{Q}$ is then given by:

\footnotetext{
${ }^{4}$ This sign as well as the plus sign in the exponent of the Bragg factor of eq. 38 and eq. 99 comes from a different convention in the time arrow between the crystallograph community and the theorists of quantum mechanics.
} 


$$
I=\frac{K}{V^{2}}\left|\sum_{a=1}^{N_{e}} \sum_{b=1}^{n_{a}} S_{b}\left(e^{i \mathbf{Q} \cdot \mathbf{R}_{a}}\left(f_{0 a}+i f_{m a}+f_{a}^{\prime}(\omega)+i f_{a}^{\prime \prime}(\omega)\right)\right)\right|^{2},
$$

where we have the non resonant Thomson $f_{0 a}$ and non resonant magnetic scattering amplitude $f_{m a}$ of the atom $a$ and the resonant terms $f_{a}^{\prime}$ and $f_{a}^{\prime \prime}$. The latter terms contain the eventual magnetic resonant contribution, sometimes written in separate terms. In the formula $K$ is a constant, $V$ the volume of the unit cell and $\mathbf{R}_{a}$ the position of the atom $a$.

In this context, during the last years there has been a strong interest in the socalled forbidden reflections which are nearly zero far from the absorption edges but with sharp intensity around the edge (as in the example of Fig. 6). These reflections are such that the sum of the Bragg terms are zero, and the intensity can be nearly proportional to $\left|f_{a}-f_{b}\right|^{2}$ where $a$ and $b$ are the two atoms possibly related by a symmetry operation (a rotation-translation or a glide plane). Thus the isotropic contributions disappear and one remains with the anisotropic ones giving the so-called Templeton effect [45]. Very weak peaks often come from a slight symmetry breaking, thus they are, let say, nearly forbidden. These reflections can give information on the change occurring during the transition between two previously equivalent atoms. In XANES one measures always a sum (and no $f^{\prime}$ neither). It is clear that the sensitivity is enhanced when measuring a difference. It is what has been used in the study of charge ordering in different compound as magnetite [38].

In Fig. 12 typical resonant scattering amplitudes for isolated atoms and for iron atoms in magnetite are shown. The relative amplitude of these terms with the Thomson scattering amplitude (which is typically equal to the atomic number) shows the importance of the anomalous effect around the absorption edge. The effect of the surrounding atoms is shown when comparing the atomic and the atom embedded in real structure spectra. For magnetite there are two different iron sites, one in octahedral environment, the other in tetrahedral environment. They have also very different shapes. Note also that their $f^{\prime \prime}$ is typical of the XANES spectra shapes.

Relation with the density of states

With the same notation, but without spin for simplicity, the density of states (or its projection on the atom) is given by:

$$
\rho_{\ell, m}^{\ell^{\prime}, m^{\prime}}(r, E)=\left|\sum_{f} a_{\ell, m}^{f}(E) a_{\ell^{\prime}, m^{\prime}}^{f *}(E) \bar{b}_{\ell}(r, E) \bar{b}_{\ell^{\prime}}^{*}(r, E)\right| .
$$

One can integrate the radial part up to the atom radius and we get:

$$
n_{\ell, m}^{\ell^{\prime}, m^{\prime}}(r, E)=\left|\sum_{f} a_{\ell, m}^{f}(E) a_{\ell^{\prime}, m^{\prime}}^{f *}(E)\right| R_{\ell, \ell^{\prime}}(E)
$$

where $R_{\ell, \ell^{\prime}}(E)$ is the radial integral: 

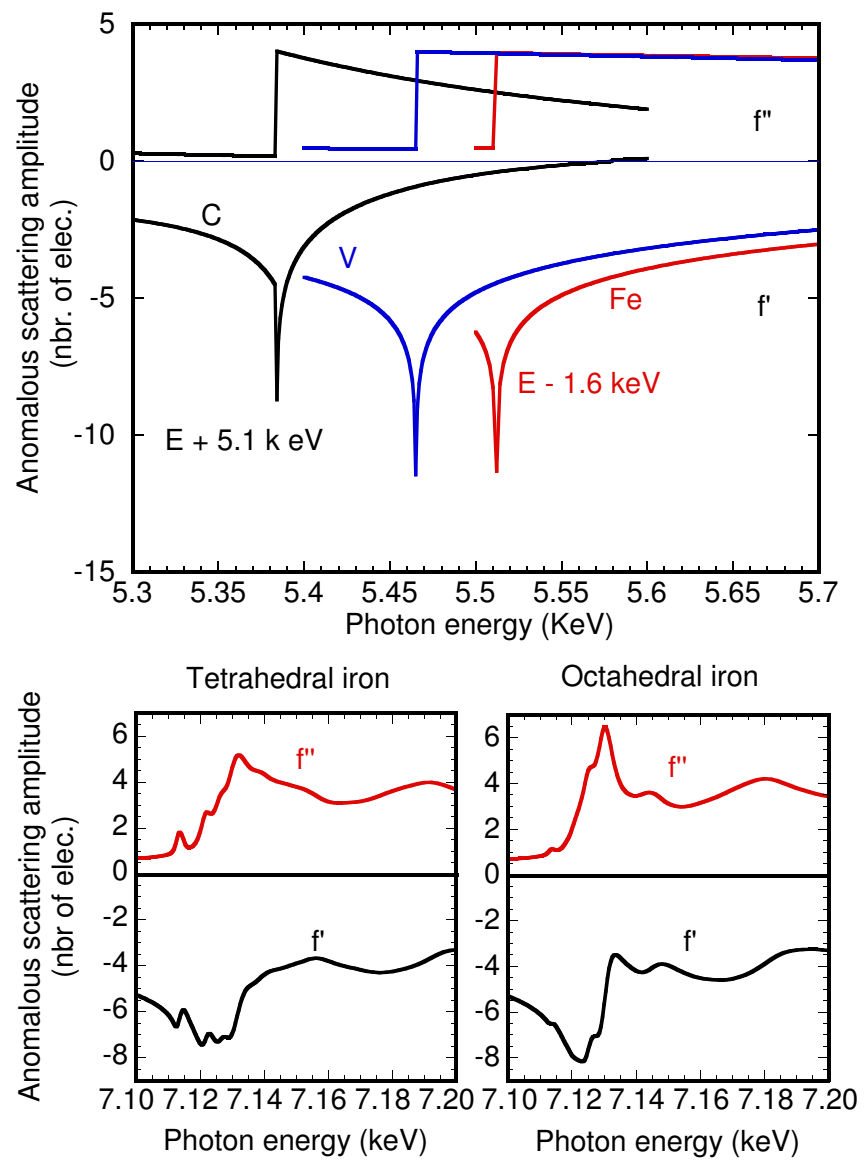

Fig. 12 Anomalous scattering amplitudes. Top: atomic spectra for carbon, vanadium and iron atoms. Bottom spectra for the iron atom in its octahedral and tetrahedral sites in magnetite at room temperature. The imaginary parts, $f^{\prime \prime}$, of the amplitudes have shapes similar to the XANES. The spectra are calculated using the FDMNES code [29].

$$
R_{\ell, \ell^{\prime}}(E)=\int_{0}^{R}\left|\bar{b}_{\ell}(r, E) \bar{b}_{\ell^{\prime}}^{*}(r, E) 4 \pi r^{2} d r\right|
$$

The diagonal term is the number of electron per unit of energy in the atom:

$$
n_{\ell, m}(E)=\sum_{f}\left|a_{\ell, m}^{f}(E)\right|^{2} R_{\ell, \ell}(E)
$$

The formulas for the absorption cross-section and for RXS contain the same $a_{\ell, m}^{f}(E) a_{\ell^{\prime}, m^{\prime}}^{f *}(E)$ terms than in the density of states formula. The difference is in the radial integral and other constant parameters. We thus see that the expressions 
are nearly proportional in most cases, except for the radial part which depends only slightly on energy. Note also, that the proportionality coefficient is different for dipole and quadrupole transitions, the dipole one being far stronger. From all this we can confirm that the absorption spectroscopy are measurements of the projection of the density of states on the absorbing atoms. Due to the selection rules, it is even a peculiar projection of the density of states, resolved in $\ell$ and eventually also in $m$, which is measured. When playing with the polarization and sometimes with the magnetic field, more selective processes can be taken into account. A good example is the measurement of circular magnetic dichroism. What is measured is then the magnetic momentum as is seen in the Sect. 6.6.

\subsection{Multipole analysis}

We have seen in the previous section that we have access to specific projection of the density of states. The access to a particular component can be obtained by playing with the polarization and taking into account the symmetry of the material. Depending on this symmetry, the different terms can be zero or not zero. The consequence is that the measurement under specific conditions can give information on the symmetry, including the magnetic state of the material. The mathematical tools, cartesian and spherical tensor algebra, which permits to make a complete analysis of these terms are seen in the following 5 .

\subsubsection{Cartesian tensors}

We have seen that the matrix elements can be expanded into dipole and quadrupole components :

$$
\left\langle\bar{\varphi}_{f}\left|\hat{o}_{i}\right| \varphi_{g}\right\rangle=D_{i}^{f}+i \frac{k}{2} Q_{i}^{f}+\ldots
$$

The signal amplitude can thus be written as:

$$
\left\langle\varphi_{g}\left|\hat{o}_{s}^{*}\right| \bar{\varphi}_{f}\right\rangle\left\langle\bar{\varphi}_{f}\left|\hat{o}_{i}\right| \varphi_{g}\right\rangle \cong D_{s}^{f *} D_{i}^{f}+i \frac{k}{2}\left(D_{s}^{f *} Q_{i}^{f}-Q_{s}^{f *} D_{i}^{f}\right)+\frac{k^{2}}{4} Q_{s}^{f *} Q_{i}^{f}
$$

We can then introduce three cartesian tensors. The dipole-dipole or E1-E1 rank 2 tensor:

$$
D_{\alpha \beta}=\sum_{f, g}\left\langle\varphi_{g}\left|r_{\alpha}\right| \bar{\varphi}_{f}\right\rangle\left\langle\bar{\varphi}_{f}\left|r_{\beta}\right| \varphi_{g}\right\rangle
$$

where $r_{\alpha}$ and $r_{\beta}$ represent $x, y$ or $z$.

In the same way we get the dipole-quadrupole or E1-E2 rank 3 tensor and the quadrupole-quadrupole or E2-E2 rank 4 tensor:

${ }^{5}$ Using other tools, Ch. Brouder gives, in a very useful paper[10], the relation between the angular dependence of the absorption cross-section and the punctual group of the studied system 


$$
\begin{aligned}
I_{\alpha \beta \gamma} & =\sum_{f, g}\left\langle\varphi_{g}\left|r_{\alpha} r_{\beta}\right| \bar{\varphi}_{f}\right\rangle\left\langle\bar{\varphi}_{f}\left|r_{\gamma}\right| \varphi_{g}\right\rangle, \\
Q_{\alpha \beta \gamma} & =\sum_{f, g}\left\langle\varphi_{g}\left|r_{\alpha} r_{\beta}\right| \bar{\varphi}_{f}\right\rangle\left\langle\bar{\varphi}_{f}\left|r_{\gamma} r_{\delta}\right| \varphi_{g}\right\rangle .
\end{aligned}
$$

where $\alpha, \beta, \gamma$ and $\delta$ label the three directions of space in Cartesian coordinates.

With this, the signal amplitude (but the multiplicative factor in the absorption cross-section and the denominator in RXS) is given by:

$A=\sum_{\alpha, \beta} \varepsilon_{\alpha}^{s *} \varepsilon_{\beta}^{i} D_{\alpha \beta}+i \frac{k}{2} \sum_{\alpha, \beta, \gamma} \varepsilon_{\alpha}^{s *} \varepsilon_{\beta}^{i}\left(u_{\gamma}^{i} I_{\alpha \beta \gamma}-u_{\gamma}^{s} I_{\alpha \beta \gamma}^{*}\right)+\frac{k^{2}}{4} \sum_{\alpha, \beta, \gamma, \delta} \varepsilon_{\alpha}^{s *} \varepsilon_{\beta}^{i} u_{\gamma}^{s} u_{\delta}^{i} Q_{\alpha \beta \gamma \delta}$.

To fix the idea the E1-E1 amplitude is given by:

$$
A_{E 1 E 1}=\left(\varepsilon_{x}^{s *}, \varepsilon_{y}^{s *}, \varepsilon_{z}^{s *}\right)\left(\begin{array}{ccc}
D_{x x} & D_{x y} & D_{x z} \\
D_{y x} & D_{y y} & D_{y z} \\
D_{z x} & D_{z y} & D_{z z}
\end{array}\right)\left(\begin{array}{c}
\varepsilon_{x}^{i} \\
\varepsilon_{y}^{i} \\
\varepsilon_{z}^{i}
\end{array}\right) \text {. }
$$

The matrix is hermitic $\left(D_{\alpha \beta}=D_{\beta \alpha}^{*}\right)$ and off-diagonal elements are complex when the material is magnetic. For example, the $D_{x x}$ term is proportional to the absorption cross-section when the polarization is along $x$.

The E1-E2 terms can be observed only for peculiar symmetry. We can remark that, for a same final state $f$, one has a product of a dipole and a quadrupole term. Due to the different selection rules ( $\Delta \ell$ odd for the first and $\Delta \ell$ even for the second), this means that $f$ must have at the same times odd and even components. In other words $f$ must be an hybridized state between odd and even components. This is possible only when the atom is not at a center of symmetry. In absorption, because of the sum on the equivalent atoms, it is sufficient to have a center of symmetry anywhere (and not necessarily on the atom) to make the E1-E2 signal zero. In RXS, on the contrary, for some reflections such that the Bragg factor is opposite on two equivalent atoms related by inversion, the signal can be observed only for the E1-E2 term, the E1-E1 and E2-E2 contributions being zero. When there is no center of symmetry, the signal can also be observed using the natural circular dichroism [37, 24]. The subtraction between the spectra obtained with the left and right polarized light eliminates the E1-E1 and E2-E2 contributions as in the diffraction technique given above. In his chapter, A. Rogalev develops examples of optical activities using the E1-E2 process (see also Ref. [42]).

\subsubsection{Spherical tensors}

The use of cartesian tensor is rather natural. Nevertheless the spherical tensors are often preferred. The reason is that their components can be related with specific observable values as for example, the orbital occupancy, the magnetic moment or the toroidal moment. These tensors are also separated in the E1-E1, E1-E2 and E2- 
E2 contributions. The amplitude can be written as:

$$
\begin{aligned}
A & =\sum_{\substack{0 \leq \ell \leq 2 \\
-\ell \leq m \leq \ell}}(-1)^{\ell+m} T_{\ell}^{m} D_{\ell}^{m}+i \sum_{\substack{1 \leq \ell \leq 3 \\
-\ell \leq m \leq \ell}}(-1)^{\ell+m} U_{\ell}^{m} I_{\ell}^{m} \\
& +\sum_{\substack{0 \leq \ell \leq 4 \\
-\ell \leq m \leq \ell}}(-1)^{\ell+m} V_{\ell}^{m} Q_{\ell}^{m},
\end{aligned}
$$

where $D_{\ell}^{m}, I_{\ell}^{m}$ and $Q_{\ell}^{m}$ are the components of the absorption-scattering tensors and $T_{\ell}^{m}, U_{\ell}^{m}$ and $V_{\ell}^{m}$ are the same for the polarization - wave vectors.

All these spherical coefficients are related to the cartesian ones. For example for the E1-E1 tensor the relations are:

$$
\begin{array}{ll}
D_{0}^{0}=\frac{1}{\sqrt{3}}\left(D_{x x}+D_{y y}+D_{z z}\right), & D_{1}^{0}=-\frac{i}{\sqrt{2}}\left(D_{x y}-D_{y x}\right), \\
-D_{1}^{1}=-\frac{i}{\sqrt{2}}\left(D_{y z}-D_{z y}\right), & D_{1}^{1}=\frac{1}{\sqrt{2}}\left(D_{x z}-D_{z x}\right), \\
D_{2}^{0}=\frac{i}{\sqrt{6}}\left(2 D_{z z}-D_{x x}-D_{y y}\right), & D_{2}^{1}=-\frac{1}{\sqrt{2}}\left(D_{x z}+D_{z x}\right), \\
+ & - \\
D_{2}^{1}=-\frac{i}{\sqrt{2}}\left(D_{y z}+D_{z y}\right), & D_{2}^{2}=\frac{i}{\sqrt{2}}\left(D_{x y}+D_{y x}\right), \\
+ &
\end{array}
$$

where we have used for all the tensors the transformation:

$$
\stackrel{ \pm}{T_{\ell}^{m}}=\frac{1}{\sqrt{2}}\left(T_{\ell}^{m} \pm T_{\ell}^{-m}\right),
$$

which gives:

$$
T_{\ell}^{m} D_{\ell}^{m}=T_{\ell}^{+}{ }^{+} D_{\ell}^{m}-\bar{T}_{\ell}^{m} \overline{D_{\ell}^{m}}
$$

The $\ell=1$ components are directly proportional to the magnetic moment vector per unit energy, $\mathbf{m}$ :

$$
\mathbf{m}=\left(\begin{array}{c}
- \\
D_{1}^{1} \\
+ \\
i D_{1}^{1} \\
D_{1}^{0}
\end{array}\right) .
$$

where we omitted the proportionality coefficient. To get the polarization spherical tensor one first writes the polarization in the matrix form :

$$
T=\left(\begin{array}{ccc}
\varepsilon_{x}^{s *} \varepsilon_{x}^{i}, & \varepsilon_{x}^{s *} \varepsilon_{y}^{i}, & \varepsilon_{x}^{s *} \varepsilon_{z}^{i} \\
\varepsilon_{y}^{s *} \varepsilon_{x}^{i}, & \varepsilon_{y}^{s *} \varepsilon_{y}^{i}, & \varepsilon_{y}^{s *} \varepsilon_{z}^{i} \\
\varepsilon_{z}^{s *} \varepsilon_{x}^{i}, & \varepsilon_{z}^{s *} \varepsilon_{y}^{i}, & \varepsilon_{z}^{s *} \varepsilon_{z}^{i}
\end{array}\right) .
$$


The spherical components of the polarization are then calculated with the same transformation than in equation 112. For example:

$$
\begin{aligned}
& T_{0}^{0}=\frac{1}{\sqrt{3}}\left(\varepsilon_{x}^{s *} \varepsilon_{x}^{i}+\varepsilon_{y}^{s *} \varepsilon_{y}^{i}+\varepsilon_{z}^{s *} \varepsilon_{z}^{i}\right)=\frac{1}{\sqrt{3}} \varepsilon_{\mathbf{s}} \cdot \varepsilon_{\mathbf{i}}, \\
& T_{1}^{0}=-\frac{i}{\sqrt{2}}\left(\varepsilon_{x}^{s *} \varepsilon_{y}^{i}-\varepsilon_{y}^{s *} \varepsilon_{x}^{i}\right)=-\frac{i}{\sqrt{2}}\left(\varepsilon_{\mathbf{s}}^{*} \times \varepsilon_{\mathbf{i}}\right)_{z} .
\end{aligned}
$$

Using all these relations one gets for the E1-E1 signal:

$A_{E 1 E 1}=\frac{1}{3} \varepsilon_{\mathbf{s}} \cdot \varepsilon_{\mathbf{i}} \operatorname{Tr}(D)-\frac{i}{\sqrt{2}}\left(\varepsilon_{\mathbf{s}}^{*} \times \varepsilon_{\mathbf{i}}\right) \cdot \mathbf{m}+T_{2}^{0} D_{2}^{0}-\stackrel{+}{T_{2}^{1}}+\stackrel{+}{D_{2}^{1}}+\overrightarrow{T_{2}^{1}}-\overrightarrow{D_{2}^{1}}+\stackrel{+}{+}+\stackrel{+}{T_{2}^{2}} D_{2}^{2}-\overrightarrow{T_{2}^{2}}-\overrightarrow{D_{2}^{2}}$.

where $\operatorname{Tr}(D)$ is the trace of $D$.

The first term in this equation does not depend on the polarization orientation in XANES because the scalar product is just one (it is also the case in RXS when keeping the relative orientation of $\varepsilon_{\mathbf{i}}$ and $\varepsilon_{\mathbf{S}}$ ). It is thus called the electric monopole component because it probes only the electronic density and not its magnetic part. It gives the E1-E1 isotropic part of the signal in the absorption cross-section and in RXS for the $\sigma-\sigma$ polarization (there is also an E2-E2 isotropic part). It is the main contribution in the XANES cross-section. When the final states are rather localized, one gets the so-called white line at the beginning of the edge. Because the electric monopole measures the charge of the non occupied states, the surface of this white line is roughly proportional to the number of hole in the corresponding state of the atom (for example the $3 d$ state in a $\mathrm{L}_{I I, I I I}$ edge). By difference, one thus can get an idea of the electron occupancy rate in the atom: larger is the white line, less electrons are in the atom ${ }^{6}$.

The second term is strictly magnetic. One sees that it is proportional to the magnetic moment through a scalar product with $\varepsilon_{\mathrm{s}}^{*} \times \varepsilon_{\mathbf{i}}$. It can thus be probed with circular polarization. The last terms are the electric quadrupole components. They are, as the first term, purely electric. They measure the anisotropy of the electric cloud around the absorber and are responsible of the main part of the linear dichroism (see Fig. 4). A smaller part in the pre-edge region can comes from the E2-E2 contribution. Note that the present terms, magnetic dipole and electric quadrupole, must not be confused with the dipole and quadrupole electric transition E1 and E2.

To illustrate more this, one can have a look on three formal examples following three different punctual group on the absorbing atom. We use a polarization $\varepsilon=$ $\varepsilon_{s}=\varepsilon_{i}$, that is like in the absorption or in $\operatorname{RXS} \sigma-\sigma$.

\footnotetext{
${ }^{6}$ Note that another factor can gives an idea of the atom valence state. It is the energy shift of the edge. Indeed, when an atom is more oxidized, its electrons tend to leave the atom. Consequently the potential at the core of the atom decreases and the core level becomes deeper. The incoming photon needs thus more energy to eject the electron from its core level and the edge is shifted towards higher energy. This phenomena is stronger at the $\mathrm{K}$ edges. Typically 1 electron corresponds to a shift of several eV.
} 
$m 3 m$ symmetry $\left(O_{h}\right)$

This cubic symmetry is for instance the case of a $3 d$ atom surrounded by a non distorted octahedron. Let's put the 6 surrounding atoms at the same distance along the positive and negative directions of the 3 axis. Due to the symmetry plane perpendicular to $O x, O y$ and $O z$, the off-diagonal components of the E1-E1 Cartesian tensor are zero, because of the three-fold axis its diagonal elements are equal. The amplitudes of the signal is in this case:

$$
A_{E 1 E 1}=\varepsilon^{+}\left(\begin{array}{ccc}
D_{z z} & 0 & 0 \\
0 & D_{z z} & 0 \\
0 & 0 & D_{z z}
\end{array}\right) \varepsilon=\frac{1}{3} \varepsilon \cdot \varepsilon \operatorname{Tr}(D)=D_{z z}
$$

The signal amplitude is isotropic. It does not depend on the polarization orientation.

\section{4/mmm symmetry $\left(D_{4 h}\right)$}

This symmetry can be obtained for instance by a single elongation of the previous octahedron along the $z$-axis. There is no more the three-fold axis but a four-fold axis around $z$, which makes $D_{x x}=D_{y y}$. One thus gets:

$$
A_{E 1 E 1}=\varepsilon^{+}\left(\begin{array}{ccc}
D_{x x} & 0 & 0 \\
0 & D_{x x} & 0 \\
0 & 0 & D_{z z}
\end{array}\right) \varepsilon=\frac{1}{3}\left(2 D_{x x}+D_{z z}\right)+\frac{1}{3}\left(D_{z z}-D_{x x}\right)\left(3\left|\varepsilon_{z}\right|^{2}-1\right)
$$

When using the spherical coordinates for the linear polarization :

$$
\varepsilon=\left(\begin{array}{c}
\sin \theta \cos \phi \\
\sin \theta \sin \phi \\
\cos \theta
\end{array}\right)
$$

we see that one gets a polarization orientation dependence equivalent to the harmon$\operatorname{ics} Y_{2}^{0}=\sqrt{\frac{5}{16 \pi}}\left(3 \cos ^{2} \theta-1\right)$. The signal is not anymore isotropic.

$4 / m^{\prime} m^{\prime} m$ symmetry

Let us see now a magnetic case with spin-orbit. Starting again from the non distorted octahedron, one supposes a magnetic moment along the $z$ axis on the central atom. The diagonal three-fold axis are broken as in the previous example. Due to the magnetic moment, the symmetry planes perpendicular to $O x$ and $O y$ are now multiplied by time reversal, thus one gets the $4 / \mathrm{m}^{\prime} \mathrm{m}^{\prime} m$ symmetry. The Cartesian tensor contains now off-diagonal imaginary terms which are proportional to the magnetic moment per energy unit: 


$$
\begin{aligned}
A_{E 1 E 1} & =\varepsilon^{+}\left(\begin{array}{ccc}
D_{x x} & i D_{x y}^{i} & 0 \\
-i D_{x y}^{i} & D_{x x} & 0 \\
0 & 0 & D_{z z}
\end{array}\right) \varepsilon \\
& =\frac{1}{3}\left(2 D_{x x}+D_{z z}\right)-\frac{i}{\sqrt{2}} \varepsilon^{*} \times \varepsilon \cdot \mathbf{m}+\frac{1}{3}\left(D_{z z}-D_{x x}\right)\left(3\left|\varepsilon_{z}\right|^{2}-1\right) .
\end{aligned}
$$

We get a new magnetic term. This one can be directly measured with x-ray circular dichroism, using the polarizations:

$$
\varepsilon=\frac{1}{\sqrt{2}}\left(\begin{array}{c}
1 \\
\pm i \\
0
\end{array}\right)
$$

such that:

$$
A_{E 1 E 1}^{+}-A_{E 1 E 1}^{-}=D_{x y}^{i}=m_{z} .
$$

For linear polarization one gets the same angular dependence than with the $4 / \mathrm{mmm}$ symmetry but probably with a smaller amplitude. This term reveals a non spherical electric cloud. It is not magnetic. Nevertheless it is present even without elongation of the $z$ axis. Its presence comes from the spin-orbit coupling which makes that the orbital electric cloud tends to align along the spin direction. It can then be said that, though electric, it is an indirect signature of the magnetic moment. Note that when there is also an elongation along $z$, this anisotropy reveals both phenomena together. All this means that such a linear measurement cannot be considered as a proof of a magnetization. One really needs circular polarization.

The expansion in spherical tensors can be performed also for the E1-E2 and E2E2 terms. All the components have a peculiar physical significance. They are classified in Table 3. The different terms change in sign with time reversal when they are magnetic and with inversion when they are E1-E2. This allows peculiar measurements on specific reflections or/and with peculiar polarization conditions. Time reversal can be obtained when inverting a magnetic field making one measurement with one way and another one in the other way. The different contributions can be often measured separately playing with the incoming (and outgoing in RXS) polarizations, the choice of the reflections in RXS, the way of the magnetic field and the orientation of the sample.

To finish this part we recall that the magnetic dipole transition M1 term can also exist. Thus one can get M1-M1 or E1-M1 components. These ones are nevertheless necessary very small when not zero. In the X-ray range definite proof of their measurement remains to be done.

\subsection{X-ray magnetic circular dichroism}

When making the difference between spectra recorded with left and right circular polarization one measures the circular dichroism. In the x-ray range and applied 
Table 3 List of the spherical tensor components with some of their physical significance. The couple of signs,,+++--+ and -- gives the change in sign under time reversal for the first and inversion for the second. The magnetic terms are odd by time reversal. The E1-E2 components are odd by inversion. The electric monopole term measures the charge density $c h_{\ell+1}$ in E1-E1 and a part of it $c h_{\ell+2}$ for E2-E2. The magnetic dipole terms measure the moment density $m_{\ell+1}$ in E1-E1 and a part of it $m_{\ell+2}$ for E2-E2. The magnetic dipole E1-E2 measures the toroidal moment (or anapole) $t$. The electric quadrupole E1-E2 measures the toroidal axis $(t, m)$. Other components measure higher order of electric charges or magnetic moments.

\begin{tabular}{llllll}
\hline$\ell$ & & E1-E1 & E1-E2 elec. & E1-E2 magn. & E2-E2 \\
\hline 0 & monopole & $++c h_{\ell+1}$ & & & $++\approx c h_{\ell+2}$ \\
1 & dipole & $-+m_{\ell+1}$ & $+-n$ & $--t$ & $-+\approx m_{\ell+2}$ \\
2 & quadrupole & ++ & $+-(t, m)$ & $--(n, m)$ & ++ \\
3 & octupole & & $+-(n, m, m)$ & $--(t, m, m)$ & -+ \\
4 & hexadecapole & & & & ++ \\
\hline
\end{tabular}

on magnetic material, this technique is called $\mathrm{x}$-ray magnetic circular dichroism (XMCD). We have already seen in the previous chapter, that is is a way to measure the magnetic moment on the atoms. Let us see a bit more in detail how the measurements can give a quantitative evaluation of these parameters. We shall also see that using sum rules one can in many cases separate the angular and the spin momentum. The x-ray sum rules were derived at the beginning of the 90s by Thole, Carra and coworkers [46, 13] from magneto-optics sum rules. Numerous experimental and theoretical studies were aimed at investigating their validity for itinerant magnetic systems. The adequacy of the sum rules varied from very good to poor. It is with the experimental work of Chen [14] with a special care on the experimental artifacts that their validity were proved.

For the $\mathrm{L}_{I I, I I I}$ edges of the $3 d$ elements, we can use the simplified equation 97 because the quadrupolar transition is negligible. We choose polarizations such that:

$$
\varepsilon \cdot \mathbf{r}=\sqrt{\frac{4 \pi}{3}} r Y_{1}^{ \pm 1}
$$

using the complex harmonics and with $m= \pm 1$ for the left and right circular polarized light. There are 4 initial states in the $p_{3 / 2}$ configuration corresponding to the $\mathrm{L}_{I I I}$ edge and only 2 for the $p_{1 / 2}$ configuration corresponding to the $\mathrm{L}_{I I}$ edge ${ }^{7}$. From the different values of the Gaunt coefficients for the $\mathrm{L}_{I I}$ and $\mathrm{L}_{I I I}$ edges and from the different values of the $G_{g}^{\sigma}$ in the equations 66 and 67, we can show that the transition probability for the $\mathrm{XMCD}$ is not the same for the orbital and spin part in $\mathrm{L}_{I I}$ and $\mathrm{L}_{I I I}$.

We use also the fact that the $3 d$ are rather localized. This means that they spread in a small energy range and thus integration in an energy range containing all the

\footnotetext{
${ }^{7}$ The relative number of state (and so of electron) in the initial state, gives the often observed ratio 2 between both edges. Note also that the ratio is exactly equal to 2 when there is no multi-electronic effect and no spin-orbit in the final state. Getting the good branching ratio is one of the challenges for the multi-electronic theories
} 
empty $3 d$ states is possible. When measuring the absorption cross-section, whatever is the polarization, one probes the unoccupied states. The idea is that by integration, one measures the number of free states in electron unit, the occupied states being given by the difference with the total number of electrons per atom in a $3 d$ band, that is 10 . The difficulties is that the states spread in the continuum. The photo electron mainly probes the $3 d$ states but also all the $4 d, 5 d, 6 d$ and so on. Because we are in the continuum, $n$ is not anymore a good quantum number and the $n>3$ (and the $s$ states) must be considered as a continuous background. Thus, before making the integration, a double step like function, with each height equal to the absorption after each white line, is subtracted. For practical purposes, the integrations are performed on an range extending typically from $20 \mathrm{eV}$ before the $\mathrm{L}_{I I I}$ edge up to $40 \mathrm{eV}$ after the $\mathrm{L}_{I I}$ edge.

From all this, one gets 2 equations for the two unknowns. The normalization is performed with the absorption edge which is, as we know, a measurement of the density of states. At the end one gets the sum rules:

$$
\begin{aligned}
m_{\text {orb }} & =-\frac{4}{3 N}\left(10-n_{3 d}\right) \int_{L_{I I}+L_{I I I}}\left(\sigma^{+}-\sigma^{-}\right) d E \\
m_{\text {spin }} & =-\frac{2}{N}\left(10-n_{3 d}\right)\left(1+\frac{7\left\langle T_{z}\right\rangle}{2\left\langle S_{z}\right\rangle}\right)^{-1} \\
& \times\left(3 \int_{L_{I I I}}\left(\sigma^{+}-\sigma^{-}\right) d E-2 \int_{L_{I I}+L_{I I I}}\left(\sigma^{+}-\sigma^{-}\right) d E\right)
\end{aligned}
$$

with the normalization :

$$
N=\int_{L_{I I}+L_{I I I}}\left(\sigma^{+}+\sigma^{-}\right) d E
$$

where $m_{\text {orb }}$ and $m_{\text {spin }}$ are respectively the orbital and spin magnetic moments in unit of $\mu_{b} /$ atom. $n_{3 d}$ is the $3 d$ electron occupation number of the specific transition metal atom. The $\mathrm{L}_{I I}$ and $\mathrm{L}_{I I I}$ denote the integration range. $\left\langle T_{z}\right\rangle$ is the expectation value of the magnetic dipole operator and $\left\langle S_{z}\right\rangle$ is equal to $m_{s p i n}$ in Rydberg atomic units. The ratio $\left\langle T_{z}\right\rangle /\left\langle S_{z}\right\rangle$ can be estimated by first principal calculation but for practical purposes, it is only some percents and thus this term can often be neglected. On the contrary one has to make an estimation for $n_{3 d}$. These sum rules are sufficient to extract, experimentally, the $m_{\text {orb }}$ and $m_{\text {spin }}$ values.

\section{Mono electronic simulations}

As stated above simulations can be very helpful in the interpretation of the experiments. Moreover, in order to quantitatively access to parameters such as atom positions, charges or magnetic moments, they can be absolutely necessary. We have seen that in some cases ligand field multiplet theory is necessary. This point is developed 
by F. de Groot in another chapter. Here we focus on the mono electronic simulations. They often gives satisfactory results for the K-edges and the $\mathrm{L}_{I I, I I I}$ edges of heavy elements.

The mono electronic simulations uses the local (spin) density approximation (L(S)DA) of the density functional theory (DFT). There are two groups of calculation method: the one solving the Schrödinger (or Dyson, or Dirac) equation in a cluster centered around the absorbing atom and the one, usually derived from band structure calculations, using the 3D periodicity of the material. The calculations can also be performed with or without self-consistency.

Whatever the method is, the first thing we need is a potential. It is seen briefly in the next section. About the different methods of calculation (Linear augmented plane wave (LAPW) [5], tight-banding, linear combination of atomic orbital (LCAO), pseudo-potential [12], linear muffin-tin orbital (LMTO), KKR [22], finite difference...) the most used for practical purposes is the multiple scattering theory (MST) that is discussed a bit more in Sect. 7.2. At the end, a table shows the different codes available.

\subsection{The potential}

It is often said that all the methods are equivalent, at the end. Although it might be true, in fact, they give different results... This is due to the fact that, inside each method, approximations are done. Expansion in spherical harmonic or in plane waves are limited, there are interpolations in the building of the potential, calculations are in single or double precision, but in particular, there are the potential problems. The first one comes from the approximation done on the potential shape. The second is related to the choice of the exchange correlation potential.

The multiple scattering theory, as the LMTO, usually makes an approximation on the potential shape. To have the calculation simpler, the potential is taken as spherically symmetric in the atoms and constant between them, in the interstitial region. This is the so-called muffin-tin approximation. The radius of the atoms (of the spherical part) is thus a technical difficulty, each code author having its own recipe. Most often a small overlap is authorized because, pragmatically, this improves the agreement with the experiments. Nevertheless from a mathematical point of view this trick is not justified. Now, using the finite difference method, pseudo potential or FLAPW technique for example, it is possible to have free-shape (or full) potential. The price to pay is that calculations are heavier. Nevertheless when the structures are sparse, when there are few symmetry elements or when the absorbing atom is relatively light, important differences are observed due to the muffin-tin approximation [29]. A recent work has nevertheless shown that the multiple scattering theory, up to now always using the muffin-tin approximation, can also be applied with a full potential shape [26].

In the LSDA the exchange-correlation problem is treated with a local potential which depends only on the local density of electrons (that is, on the point where one 
calculates the potential). In the magnetic case it depends as well on the local difference between the spin-up and spin-down density. This one can have different forms. Presently the most used ones are the Hedin and Lundqvist [27] and the Perdew and Wang [39]. Globally they give an attractive potential of increasing amplitude with increasing electron density. For XAS it is important to consider the energy dependence of this potential, as proposed by Von Bart and Hedin [48]. Indeed the amplitude of the potential decreases with increasing electron kinetic energy. In a relatively narrow energy range around the plasmon energy, that is between 10 and $30 \mathrm{eV}$, this potential changes by several $\mathrm{eV}$. Without considering this phenomena one gets structures in the spectra shifted by the same amount. Because the position of the oscillations are related to the inter atomic distances, this could lead to false agreement with wrong fit of the corresponding parameters.

\subsection{The multiple scattering theory}

Explain this theory in a single paragraph is difficult. Readers can find detailed description by Natoli and coworkers [35] or Brouder [11]. There are two ways to explain it. The first way uses the Green's function approach, the second uses the scattering wave approach. Let us use the second one.

First, one considers just one atom. We build a complete basis in the surrounding vacuum. There, the potential is constant and the solutions of the radial Schrödinger equation are the Bessel, $j_{\ell}$, Neuman and Hankel functions. Using the phase shift theory one looks how the atom scatters all the Bessel functions. One uses the continuity of the wave function and its derivative at the border. For simplicity we keep the non magnetic case, and we can write:

$$
a_{\ell}(k) b_{\ell}(k, R) Y_{\ell}^{m}=\sqrt{\frac{k}{\pi}}\left(j_{\ell}(k R)-i t_{\ell} h_{\ell}^{+}(k R)\right) Y_{\ell}^{m},
$$

for the wave function at the muffin-tin radius $R . h_{\ell}^{+}(r)$ is the Hankel outgoing function, $t_{\ell}$ is the atomic scattering amplitude, $b_{\ell}$ the solution of the radial Schrödinger equation in the atom, $k$ the electron wave vector and $a_{\ell}$ the amplitude inside the atom. All these terms depend on the kinetic electron energy. We have introduced the normalization by the density of states in vacuum $\sqrt{\frac{k}{\pi}}$. By continuity this normalization makes that the density of states is included in the atomic amplitudes. Using the two equations of continuity (function and derivative), one gets $a_{\ell}$ and $t_{\ell}$.

Now the atom is embedded in a cluster. Thus the incoming wave is not anymore included in a simple Bessel function, but the superposition of this Bessel function and all the other waves of Hankel type, backscattered by the other atoms. The problem is thus not anymore spherical, and the scattering and atomic amplitudes will also depend on the quantum number $m$ (and eventually on the spin index). One has to consider all the scattering processes from one atom to any other atom. To do that, one fills a (big) multiple scattering matrix, containing, for all the atoms, all 
their individual expansion in spherical harmonics. Its diagonal contains the atomic scattering amplitudes. The off-diagonal part contain the propagation terms connecting the scattering from the $(\ell, m)$ of an atom $a$, to the $\left(\ell^{\prime}, m^{\prime}\right)$ of another atom $a^{\prime}$. The inversion of this matrix gives the multiple scattering amplitudes, $\tau_{\ell, m, a}^{\ell^{\prime}, m^{\prime}, a^{\prime}}$, by the relation:

$$
\tau_{\ell, m, a}^{\ell^{\prime}, m^{\prime}, a^{\prime}}=\left[\frac{1}{1-T H} T\right]_{\ell, m, a}^{\ell^{\prime}, m^{\prime}, a^{\prime}} .
$$

$T$ is a diagonal matrix containing the atomic scattering amplitudes. $H$ is the matrix containing the propagation terms.

Most of the computing time resides in the inversion of this matrix. When we are not interested in the low photo-electron energy range, it is possible to perform a Taylor expansion (this is the so-called path expansion):

$$
\tau_{\ell, m, a}^{\ell^{\prime}, m^{\prime}, a^{\prime}} \cong\left[T+T H T+(T H)^{2} T+(T H)^{3} T+\ldots\right]_{\ell, m, a}^{\ell^{\prime}, m^{\prime}, a^{\prime}},
$$

where the number of paths increases with the power of $T H$. The limitation of this number is one of the key point in the IFEFfit [28] and GNXAS [18] codes. At the rising edge it is not possible to perform this path expansion, because terms in the denominator are bigger than 1 and the series never converge [36].

Using $a=a^{\prime}$ and skipping the atom index, the optical theorem gives:

$$
-\mathfrak{I}\left(\tau_{\ell, m}^{\ell^{\prime}, m^{\prime}}\right)=\left|\sum_{f} a_{\ell, m}^{f *} a_{\ell^{\prime}, m^{\prime}}^{f}\right| .
$$

when the potential is real. Introducing this in the XANES formula, one gets :

$$
\sigma=-4 \pi \alpha \hbar \omega \sum_{g} \sum_{\ell, m, \ell^{\prime}, m^{\prime}} \mathfrak{I}\left(\left\langle\varphi_{g}\left|\hat{o}^{*}\right| \bar{b}_{\ell} Y_{\ell}^{m}\right\rangle \tau_{\ell, m}^{\ell^{\prime}, m^{\prime}}\left\langle\bar{b}_{\ell^{\prime}} Y_{\ell^{\prime}}^{m^{\prime}}|\hat{o}| \varphi_{g}\right\rangle\right)
$$

The central term $\left|\bar{b}_{\ell} Y_{\ell}^{m}\right\rangle \tau_{\ell, m}^{\ell^{\prime}, m^{\prime}}\left\langle\bar{b}_{\ell^{\prime}} Y_{\ell^{\prime}}^{m^{\prime}}\right|$ is the Green's function.

One can do the same for the RXS case. To be more complete, we have written the equation in the spin-orbit case:

$$
\begin{aligned}
f^{\prime}-i f^{\prime \prime} & =m \omega^{2} \\
& \times \sum_{g, \sigma} \sum_{\substack{\ell, m, s \\
\ell^{\prime}, m^{\prime}, s^{\prime}}}\left\langle\varphi_{g}^{\sigma}\left|o_{s}^{*}\right| \bar{b}_{\ell, m+\frac{1}{2}-\sigma, s}^{\sigma} Y_{\ell}^{m}\right\rangle \tau_{\ell, m+\frac{1}{2}-s, s}^{\ell^{\prime}, m^{\prime}+\frac{1}{2}-s^{\prime}, s^{\prime}}\left\langle\bar{b}_{\ell^{\prime}, m^{\prime}+\frac{1}{2}-\sigma, s^{\prime}}^{\sigma}\left|o_{i}\right| \varphi_{g}^{\sigma}\right\rangle .
\end{aligned}
$$

When the potential is complex, the expression contains more terms because the irregular solutions of the radial Schrödinger equation have to be considered, as well. 


\subsection{Available codes}

There are a number of codes which permits to perform mono-electronic simulations of the absorption spectra. We present a probably non exhaustive list of them in Table 4. Some use the 3D periodicity of the solid, some others use the cluster approach. One has first to recall that the first theories are due to Dill and Dehmer in 1974 [19] and Lee and Pendry in 1975 [31]. The first calculations using a cluster MST approach are due to Natoli et al. [35] and those using a band structure approach were first performed by Mattheiss and Dietz [34], both in 1980.

Table 4 Some of the most used mono electronic codes for absorption spectroscopy. $\mathrm{Cl}$ and 3D means respectively cluster and $3 \mathrm{D}$ approach, SCF means self-consistent calculation, Fit means there is a fit procedure with experimental spectra to get parameters. Note that a Feff version used in the intermediate energy range called IFEFfit [28] and the code GNXAS [18] use the path expansion in the MST frame and allows a fit procedure to get geometrical parameters. Cabaret et al. uses a pseudo-potential approach having modified first the PARATEC code and more recently the PWSCF code.

\begin{tabular}{|c|c|c|c|c|c|c|}
\hline Authors & Name & Technique & $3 \mathrm{D}$ & $\mathrm{SCl}$ & Fit & Note and Reference \\
\hline Natoli & CONTINUUM & MST & $\mathrm{Cl}$ & & & [35] The first ! \\
\hline Benfatto & MXAN & MST & $\mathrm{Cl}$ & & $X$ & {$[4]$} \\
\hline Rehr et al. & FEFF & MST & $\mathrm{Cl}$ & $X$ & & [1] The most used \\
\hline Joly & FDMNES & MST \& FDM & $\mathrm{Cl}$ & $X$ & $\mathrm{X}$ & [29] \\
\hline Ebert et al. & SPRKKR & KKR & $3 \mathrm{D}$ & $X$ & & {$[22]$} \\
\hline Blaha et al. & Wien2k & FLAPW & $3 \mathrm{D}$ & $\mathrm{X}$ & & [5] \\
\hline Cabaret et al. & & Pseudo & $3 \mathrm{D}$ & $\mathrm{X}$ & & [12] \\
\hline Saint-Amant et al. & StoBe & LCAO & $3 \mathrm{D}$ & $X$ & & [43] \\
\hline Vedrinskii et al. & XKDQ & MST & $\mathrm{Cl}$ & & & [47] \\
\hline Yaresko et al. & Py-LMTO & LMTO & $3 \mathrm{D}$ & $X$ & & [49] \\
\hline
\end{tabular}

\section{Conclusion}

We have reviewed the different phenomena governing the x-ray spectroscopies related to the transition of an electron from a core level up to some other level. The number of applications of these processes is huge. They permit a precise understanding of spectroscopies giving different pieces of information on the materials. Because the process of transition is complex, the interpretation is not always direct. Some of them need multiplet calculations as explained by F. de Groot in another chapter. Some others can use mono electronic simulations as presented here. Some of the spectroscopic techniques are presented in details in the following chapters of this book. These are the Inelastic X-ray Scattering by J.-P. Rueff, the X-ray detected Magnetic Resonance by J. Goulon, A. Rogalev and F. Wilhelm, Resonant X-ray 
Scattering by S. P. Collins and A. Bombardi. A. Rogalev develops also examples of optical activities.

Acknowledgements All my gratitude goes to Calogero Natoli for his constant help. Most of the community has benefited of its enormous knowledge kindly shared. I also specially thank Christian Brouder, Delphine Cabaret, Emilio Lorenzo, Stéphane Grenier, Hélio Tolentino and Didier Sebilleau for their support and their useful advices.

\section{References}

1. A.L. Ankudinov, B. Ravel, J.J. Rehr and S.D. Conradson, Phys. Rev. B 58, 7565 (1998). http://leonardo.phys.washington.edu/feff/

2. A.L. Ankudinov, A.I. Nesvizhskii, and J.J. Rehr, Phys. Rev. B 67, 115120 (2003).

3. V.V. Balashov in Interaction of Particles and Radiation with Matter, (Springer, Berlin, Heidelberg, New York, 1997), p. 136

4. M. Benfatto and S. Della Longa, J. Synchrotron Rad. 8, 1087 (2001).

5. P. Blaha, K. Schwarz, G. Madsen, D. Kvasnicka and J. Luitz in An augmented plane wave + local orbital program for calculationg crystal properties, (TU WIEN 2001).

6. M. Blume, J. Appl. Phys 57, 3615 (1985).

7. M. Blume, Doon Gibbs, Phys. Rev. B 37, 1779 (1988).

8. M. Blume, in Resonant Anomalous X-ray scattering, edited by G. Materlik, J. Sparks and K. Fisher (Elsevier, Amsterdam, 1994), p. 495.

9. S.V. Borisenko, A. A. Kordyuk, V. Zabolotnyy, J. Geck, D. Inosov, A. Koitzsch, J. Fink, M. Knupfer, B. Büchner, V. Hinkov, C. T. Lin, B. Keimer, T. Wolf, S. G. Chiuzba?ian, L. Patthey, and R. Follath Phys. Rev. Lett. 96, 117004 (2006).

10. Ch. Brouder, J. of Phys. Cond. Matt, 2, 701 (1990).

11. Ch. Brouder, in Magnetism and Synchrotron Radiation, edited by E. Beaurepaire, B. Carriére, J.-P. Kappler (Les Editions de Physiques, 1997), p. 33-54.

12. D. Cabaret, E. Gaudry, M. Taillefumier, P. Sainctavit and F. Mauri Phys. Scripta T115, 131 (2005).

13. P. Carra and M. Altarelli, Phys. Rev. Lett. 64, 1286 (1990).

14. C.T. Chen, Y.U. Idzerda, H.-J. Lin, N.V. Smith, G. Meigs, E. Chaban, G.H. Ho, E. Pellegrin and F. Sette, Phys. Rev. Lett. 75, 152 (1995).

15. C. Cohen-Tannoudji, J. Dupont-Roc, G. Grynberg, in Processus d'interaction entre photons et atomes, Chapter 2 (InterEditions/Editions du CNRS, Paris 1988).

16. D.C. Creagh, in International Tables for Crystallography, ed. by International Union of Crystallography, vol C, Chapter 4.1 (Springer, Heidelberg, 2006), p. 186

17. F. de Bergevin, M. Brunel, Acta Cryst. A37, 314 (1981).

18. http://gnxas.unicam.it/

19. Dan Dill and J.L. Dehmer, J. of Chem. Phys. 61, 692 (1974).

20. Ch. Den Auwer, private communication.

21. S. Di Matteo, Y. Joly, and C. R. Natoli, Phys. Rev. B 72, 144406 (2005).

22. H. Ebert, in Electronic Structure and Physical Properties of Solids, ed. (Springer Berlin, 2000), p. 191.

23. R.-M. Galéra, Y. Joly, A. Rogalev and N. Bingelli, J. Phys.: Condens. Matter 20, 395217 (2008).

24. J. Goulon, C. Goulon-Ginet, A. Rogalev, V. Gotte V, C. Malgrange, Ch. Brouder, C. R. Natoli J. of Chem. Phys., 108, 6394 (1998).

25. S. Grenier, A. Toader, J. E. Lorenzo, Y. Joly, B. Grenier, S. Ravy, L. P. Regnault, H. Renevier, J. Y. Henry, J. Jegoudez and A Revcolevschi, Phys. Rev. B 65, 180101 (2002). 
26. K. Hatada, K. Hayakawa, M. Benfatto and C.R. Natoli, Phys. Rev. B 76, 060102 (2007).

27. L. Hedin and S. Lundqvist, J. Phys. C: Solid State Phys. 4, 2064 (1971).

28. http://cars9.uchicago.edu/ifeffit/

29. Y. Joly, Phys. Rev. B 63, 125120 (2001). http://www.neel.cnrs.fr/fdmnes.

30. P. Kruger and C. R. Natoli, Phys. Scripta T115, 146 (2005).

31. P.A. Lee and J.B. Pendry, Phys. Rev. B 11, 2795 (1975).

32. P.A. Lee, Phys. Rev. B 13, 5261 (1976).

33. M. A. L. Marques, C. A. Ullrich, F. Nogueira, A. Rubio, K. Burke, E. K. U. Gross, in TimeDependent Functional Theory, Lect. Notes Phys. 706, ed. (Springer Berlin Heidelberg, 2006).

34. L.F. Mattheiss and R.E. Dietz, Phys. Rev. B 22, 1663 (1980).

35. C. R. Natoli, D. K. Misemer, S. Doniach and F. W. Kutzler, Phys. Rev. A 22, 1104 (1980).

36. C. R. Natoli, M. Benfatto, J. de Phys. C 8, 11 (1986).

37. C. R. Natoli, Ch. Brouder, Ph. Sainctavit, J. Goulon, C. Goulon-Ginet, A. Rogalev, Eur. Phys. J. B,4, 1 (1998).

38. E. Nazarenko, J.E. Lorenzo, Y. Joly, J.-L. Hodeau, D. Mannix, and C. Marin, Phys. Rev. Lett. 97, 056403 (2006)

39. J. P. Perdew and Y. Wang, Phys. Rev. B 45, 13244 (1992).

40. B. Poumellec, R. Cortes, G. Tourillon, J. Berthon, Phys. Stat. Sol. (b) 64, 319 (1991).

41. O. Proux and J.-L. Hazemann, measured at the FAME beam line at the ESRF, private communication.

42. A. Rogalev, J. Goulon, F. Wilhelm, Compte rendu Physique, 9, 642 (2008).

43. http://w3.rz-berlin.mpg.de/ hermann/StoBe/

44. J.A. Soininen and E.L. Shirley, Phys. Rev. B 64, 165112 (2001).

45. D. H. Templeton, and L. K. Templeton, Acta Cryst. A41, 365 (1985).

46. B.T. Thole, P. Carra, F. Sette and G. van der Laan, Phys. Rev. Lett. 68, 1943 (1992).

47. R.V. Vedrinskii, V.L. Kraizman and A.A. Novakovich, Phys. Status Solidi B 226, 203 (2001).

48. I. U. Von Barth and L. Hedin, J. Phys. C: Solid State Phys. 5, 1629 (1972).

49. A.N. Yaresko, V.N. Antonov, H. Eschrig, P. Thalmeier and P. Fulde, Phys. Rev. B 62, 15538 (2003). 